\title{
Market capitalization and Value-at-Risk
}

\author{
Alexandra Dias* \\ School of Management \\ University of Leicester, UK
}

\author{
Final draft \\ Journal of Banking and Finance, 37(12), 2013 \\ DOI: $10.1016 /$ j.jbankfin.2013.04.015
}

\begin{abstract}
The potential of economic variables for financial risk measurement is an open field for research. This article studies the role of market capitalization in the estimation of Value-at-Risk (VaR). We test the performance of different VaR methodologies for portfolios with different market capitalization. We perform the analysis considering separately financial crisis periods and non-crisis periods. We find that VaR methods perform differently for portfolios with different market capitalization. For portfolios with stocks of different sizes we obtain better VaR estimates when taking market capitalization into account. We also find that it is important to consider crisis and non-crisis periods separately when estimating VaR across different sizes. This study provides evidence that market fundamentals are relevant for risk measurement.
\end{abstract}

${ }^{*}$ Correspondence address: Alexandra Dias, School of Management, Ken Edwards Building, University of Leicester, LE1 7RH Leicester, UK. Tel: +44(0)116 252 5520. Fax: +44(0)116 2523949. E-mail: Alexandra.Dias@le.ac.uk 
Keywords: Market capitalization, quantitative risk management, Value-at-Risk, financial crises.

JEL classification: C5, C21, G01, G17, G28, G32 


\section{Introduction}

The accords on banking supervision from the Bank for International Settlements (BIS) single out Value-at-Risk (VaR) as a measure of financial risk. The BIS accords and in particular VaR play a central role in financial risk measurement and management. Despite its importance the most popular methods in practice for estimating VaR (historical simulation and RiskMetrics) are yet relatively simple. This is a constraint of real-world. The complexity of financial institutions calls for sound simple models, easy to estimate.

There is a vast academic literature on methods for estimating VaR. These methods can be very sophisticated and they are mainly reduced form in the sense that they explain risk in an autoregressive manner. The very well known GARCH model (Bollerslev (1986)) is perhaps the prime example of this. Recently there has been an increased interest on structural approaches for risk measurement involving market and macro-economic variables; see Andersen et al. (2012) for a detailed overview. This article contributes to this stream of research. Our aim is to understand the relation between stock size, measured by market capitalization, and equity risk measured by VaR.

By definition the $100 \alpha \%$ VaR is the value such that the probability of observing a loss larger than VaR is smaller than the confidence level $1-\alpha$, over a given time horizon. The time horizon usually is a 1-day or 10-day holding period for market risk and 1 year for credit and operational risk. The confidence level $\alpha$ typically ranges between $95 \%$ and $99 \%$. Hence VaR is in the tail of the profit-and-loss or returns distribution. This 
fact makes the estimation of VaR a difficult task. In probabilistic terms the definition of $\mathrm{VaR}$ is very simple. $\mathrm{VaR}$ is the negative of the $1-\alpha$ probability quantile of the returns distribution.

To compute VaR by the existing models it is necessary to obtain an estimate of the distribution of the portfolio returns sometime in the future. The only exception is the regression quantile method introduced in Chernozhukov and Umantsev (2001) and Engle and Manganelli (2004), where the quantile of the distribution is modelled directly. All the other VaR models use different approaches to estimating the distribution of the returns. We can classify these VaR models as follows: Historical simulation, introduced by Boudoukh et al. (1998), uses the empirical distribution function obtained from past data to estimate $\mathrm{VaR}$ as an empirical quantile; Filtered historical simulation estimates VaR as an empirical quantile of the residuals obtained from fitting a parametric model to the original returns. Most commonly the method is implemented with a GARCH type model to filter the returns as introduced by Barone-Adesi et al. (1998, 1999); Fully parametric methods which model the complete returns distribution. RiskMetrics (1996) and GARCH, from Bollerslev (1986), are prime examples of fully parametric models used for estimating VaR. Chavez-Demoulin et al. (2005) introduce a parametric sophisticated alternative to the use of GARCH as a filter based on a point process approach; Extreme Value Theory (EVT) methods model the tail of the returns distribution. The filtered EVT model, where the returns are filtered with a GARCH model, was first introduced in McNeil and Frey (2000).

Since the seminal work of Banz (1981), Stattman (1980), Rosenberg et al. (1985), and Fama and French (1992) that firm and market specific variables are known to be 
useful in explaining the expected return on stocks. Both the expected return and the quantile are characteristics of the asset return distribution. It seems pertinent to ask if there is a relationship between VaR, as a quantile, and market variables. Here we concentrate on market capitalization.

Reference papers comparing the performance of different VaR models are Bao et al. (2003, 2006), Brooks et al. (2005), Kuester et al. (2006) and Pritsker (1997). Many of the studies on the computation of VaR compare and propose different methods using data on large capitalization firms, major indices or highly traded currencies. A complete list would be long but relevant examples are: Kuester et al. (2006) who use daily returns on the NASDAQ Composite Index; McNeil and Frey (2000) do backtesting on the S\&P500 and DAX indices, BMW stock prices, US dollar-British pound exchange rate and gold prices; Mancini and Trojani (2011) use the S\&P500 index, US dollar-Japanese Yen exchange rate, Microsoft and Boeing stock prices; Bao et al. (2003) use daily returns on the S\&P500 and NASDAQ indices; Engle and Manganelli (2004) implemented their CAViaR methodology on returns of General Motors, IBM and S\&P500. Hence, there might be a bias in the results found in the literature concerning the performance of VaR estimation methods.

To the best of our knowledge market variables have not been often studied in connection with VaR estimation. The empirical study of VaR methods where we found market capitalization being used is Halbleib and Pohlmeier (2012). The authors raise the question whether market capitalization is important but the paper has a much wider focus exploring the performance of different VaR models and distributional assumptions across different estimation time windows. Although within a complex study, 
the authors find evidence that market capitalization is important for VaR estimation.

The contribution of this paper is to explore the importance of market capitalization in estimating VaR. We use returns on NYSE, AMEX and NASDAQ stocks. We specifically consider separately periods of financial crises and periods without crises, challenging the performance of methods for forecasting VaR. There is a number of methods for computing VaR. Since there is no ultimate consensus on which is the best we use several methods in our study. We calculate one period ahead (out-of-sample) sequences of VaR estimates. Then we compare the sequences of VaR estimates with the realized returns and test if the estimated VaR corresponds to the level of risk desired. The result of these tests gives the performance of the VaR methods. This is called backtesting a VaR model. We backtest VaR models on ten portfolios with different market capitalization during crisis periods and non-crisis periods. We test if the methods perform differently across different market capitalization portfolios during calm and during unstable market periods. Finally we estimate VaR for a portfolio composed of stocks of different sizes with and without taking market capitalization into account. Then we compare the performance of the two approaches to find if considering market capitalization significantly improves VaR estimation.

This article is organized as follows. In Section 2 we outline the methods for the estimation of VaR used in this study. Section 3 describes the backtesting methodology which quantifies the performance of the VaR methods. The empirical implementation of the VaR estimation methods and corresponding backtesting is reported in Section 4. In Section 5 we test the significance of market capitalization in explaining the performance of VaR methods for different stock size portfolios. We study the effectiveness of using 
market capitalization on estimating the VaR of a portfolio composed of stocks with different sizes in Section 6. Finally Section 7 synthesizes the results and presents final conclusions.

\section{VaR estimation methods used in this study}

The $100 \alpha \% \mathrm{VaR}$ is the negative of the quantile of probability $1-\alpha$ of the returns distribution. In most applications $\alpha$ varies between $95 \%$ and $99 \%$ but $\alpha$ can also take the value of $99.9 \%$ as for instance it is required for operational risk in the Basel II Accord. Formally, for a confidence level $\alpha \in(0,1)$, the $100 \alpha \%$ VaR for period $t+h$, conditional on the information available up to time $t$, is given by

$$
\operatorname{VaR}_{t+h}^{\alpha}=-Q_{1-\alpha}\left(R_{t+h} \mid \mathcal{F}_{t}\right)=-\inf \left\{r \in \mathbb{R}: P\left(R_{t+h} \leq r \mid \mathcal{F}_{t}\right) \geq 1-\alpha\right\}
$$

where $R_{t}$ is the random variable representing the return in period $t, Q_{\alpha}(\cdot)$ denotes the quantile of probability $\alpha$ and $\mathcal{F}_{t}$ represents the information available at time $t$. Estimating VaR is equivalent to estimating a quantile of the unknown distribution of returns for period $t+h$.

The methods used here for estimating VaR can be classified as historical simulation (HS), fully parametric, and (semi-parametric) extreme value theory (EVT) models. Historical simulation uses empirical quantiles obtained from (filtered or not) past data. Fully parametric models characterize the complete return distribution using a, possibly dynamic, parametric model. EVT models use a parametric family to describe the tail of the distribution while the center of the returns distribution is modeled by the empirical distribution function. 
We assume that the returns can be defined as a location scale process conditional on the set of information available at time $t$ :

$$
r_{t+h}=E\left(R_{t+h} \mid \mathcal{F}_{t}\right)+\epsilon_{t+h}=\mu_{t+h}+\sigma_{t+h} z_{t+h}
$$

where $\mu_{t+h}$ is the expected return for the period $t+h$ given the information available at time $t, \sigma_{t+h}$ is the conditional scale, $\epsilon_{t+h}$ is an error term and $z_{t+h}$ has a zero location, unit scale probability density function $f_{Z}(\cdot)$. The $100 \alpha \%$ VaR forecast for the period $t+h$ conditional on the information available at time $t$ is then

$$
\operatorname{VaR}_{t+h}^{\alpha}=-\left(\mu_{t+h}+\sigma_{t+h} Q_{1-\alpha}(Z)\right)
$$

where $Q_{\alpha}$ is the $\alpha$ quantile of $f_{Z}(\cdot)$.

Different VaR methods assume different specifications for the conditional location $\mu_{t+h}$, conditional scale $\sigma_{t+h}$, and probability density $f_{Z}(\cdot)$. An outline of the VaR methods used in this study follows.

\subsection{Historical Simulation}

The simplest method of estimating VaR (see for instance Christoffersen (2012)) is to use the empirical quantile of the return distribution. This method is usually called (see Kuester et al. (2006)) the naive historical simulation. The theoretical justification for this estimator is that if we assume that the process of the returns is stationary then the empirical distribution is a consistent estimator of the unobserved future distribution function.

In order to define the estimator consider a sample of past $\omega$ returns $\left(r_{t}, r_{t-1}, \ldots, r_{t-w+1}\right)$ and the ordered sample $\left(r_{(1)}, r_{(2)}, \ldots, r_{(\omega)}\right)$, where $r_{(1)} \leq r_{(2)} \leq \ldots \leq r_{(\omega)}$ are the so- 
called ordered statistics. The historical simulation $100 \alpha \%$ VaR for period $t+1$ is given by

$$
\widehat{\operatorname{VaR}}_{t+1}^{\alpha}=-\hat{Q}_{1-\alpha}\left(r_{t}, r_{t-1}, \ldots, r_{t-w+1}\right)=-r_{([(1-\alpha) \times \omega])}
$$

where [.] represents the integer part of a real number. As an example, if we consider a sample of return observations with size $\omega=1000$, then the $90 \%$ VaR estimate is the negative of the 100-th sample statistic, $\widehat{\operatorname{VaR}}_{t+1}^{0.9}=-r_{(100)}$.

The simplicity of the historical simulation method, although attractive from the practical point of view, raises doubts about its performance. Weaknesses and possible improvements for this method are studied in Pritsker (2006) and references therein.

A more sophisticated approach consists of using the empirical quantile of the distribution $f_{Z}(\cdot)$ in model $(2)$ and equation $(3)$. This method is know as filtered historical simulation (FHS). In this approach (2) is used to pre-filter the data and then the empirical quantile is calculated from the filtered residuals, $z_{t+1}=\left(r_{t+1}-\mu_{t+1}\right) / \sigma_{t+1}$.

\subsection{Fully parametric models}

Fully parametric models assume a parametric family for the probability distribution $f_{Z}(z)$ of $z_{t}$ in equation $(2)$. The so-called unconditional parametric VaR methods assume that $\mu_{t+h} \equiv \mu$ and that $\sigma_{t+h} \equiv \sigma$. In this case the returns have probability density $f_{Z}\left(\sigma^{-1}\left(r_{t}-\mu\right)\right)$. The unconditional estimator of the $100 \alpha \%$ VaR for period $t+1$ is

$$
\widehat{\operatorname{VaR}}_{t+1}^{\alpha}=-\left(\mu+\sigma \hat{Q}_{1-\alpha}(Z)\right)
$$

In this case the VaR is the $1-\alpha$ quantile of the distribution assumed for $Z$ adjusted for location and scale. If $F_{Z}(\cdot)$ denotes the cumulative distribution function corresponding 
to $f_{Z}(\cdot)$ then the quantile is estimated by $\hat{Q}_{1-\alpha}(z)=F_{Z}^{-1}(1-\alpha)$, where $F_{Z}^{-1}(\cdot)$ is the inverse function of $F_{Z}(\cdot)$. Most studies use a family with a continuous distribution function but if the distribution function $F_{Z}(\cdot)$ is not continuous the inverse can be replaced by the generalized inverse function. In this study we assume that $Z$ can have a Normal or a Student- $t$ distribution. These two distributions, although frequently used in modeling returns, are symmetric and there is plenty of literature reporting that the returns distribution is skewed. Hence, we also consider the EVT approach as described in the next section.

In this study conditional parametric models (conditional because location and scale in equation (2) are time varying) consider a conditional mean function of past information described by an $\operatorname{ARMA}(p, q)$ model of the form

$$
\mu_{t}=\mu+\sum_{i=1}^{p} \phi_{i}\left(r_{t-i}-\mu\right)+\sum_{j=1}^{q} \theta_{j} \epsilon_{t-j}
$$

where $\phi(z)=1-\phi_{1} z-\ldots-\phi_{p} z^{p}$ and $\theta(z)=1-\theta_{1} z-\ldots-\theta_{q} z^{q}$ have no common roots and no roots inside the unit circle. We use a time varying scale parameter following a $\operatorname{GARCH}(r, s)$ process

$$
\sigma_{t}^{2}=c_{0}+\sum_{i=1}^{r} c_{i} \epsilon_{t-i}^{2}+\sum_{j=1}^{s} d_{j} \sigma_{t-j}^{2},
$$

where $c_{0}>0, c_{i} \geq 0, d_{j} \geq 0$ as introduced in Bollerslev (1986).

\subsection{VaR estimation using Extreme Value Theory}

VaR concerns only one side of the returns distribution and more specifically the tail region of the distribution. Hence EVT is especially suited for VaR estimation. For a comprehensive overview of EVT for finance see Embrechts et al. (1997). EVT studies 
the asymptotic distributional properties of the maximum of random variables. For VaR estimation we are usually concerned with the tail of the (negative) losses, hence we apply the EVT results to the right tail of the negative of the returns distribution. In this case equation (3) becomes

$$
\operatorname{VaR}_{t+h}^{\alpha}=\mu_{t+h}+\sigma_{t+h} Q_{\alpha}(Z)
$$

where $Q_{\alpha}$ is the $\alpha$ quantile of $f_{Z}(\cdot)$.

Let $\left(X_{1}, X_{2}, \ldots, X_{T}\right)$ be a sequence of iid random variables and denote its maxima by $M_{T}=\max \left(X_{1}, X_{2}, \ldots, X_{T}\right)$. If there exist coefficients $c_{T}>0$ and $d_{T} \in \mathbb{R}$, and the limit distribution of the standardized maxima $c_{T}^{-1}\left(M_{T}-d_{T}\right)$ exists then this limit distribution has to be of the form

$$
H_{\xi}(x)= \begin{cases}\exp \left\{-(1+\xi x)^{-1 / \xi}\right\} & \text { if } \quad \xi \neq 0, \\ \exp \{-\exp (-x)\} & \text { if } \quad \xi=0\end{cases}
$$

where $1+\xi x>0$. The distribution $H_{\xi}$ is called Generalized Extreme Value Distribution $(\mathrm{GEV})$. Given a random variable $X$ with distribution function $F_{X}$, if the limit distribution of the maxima $M_{T}$ exists then $F_{X}$ belongs to the so-called Maximum Domain of Attraction (MDA) of $H_{\xi}, F_{X} \in \operatorname{MDA}\left(H_{\xi}\right)$. Most of the continuous distributions commonly used in applied statistics are in the $\operatorname{MDA}\left(H_{\xi}\right)$. See Embrechts et al. (1997) for a detailed account of the distributions in the $\operatorname{MDA}\left(H_{\xi}\right)$. The shape parameter $\xi$ controls the shape of the tail of the distribution. If $\xi<0$ the distribution of $X$ has a finite right end point, for instance a uniform distribution, and the limit distribution $H_{\xi}$ is a Weibull distribution. In case $\xi=0, H_{\xi}$ is a Gumbel distribution and the tail of the distribution of $X$ decreases at an exponential rate as it is the case of the Normal. When $\xi<0$ the distribution $F_{X}$ has a heavy tail and $H_{\xi}$ is a Fréchet distribution. The 
later is the most useful case in finance as returns very often have a heavy tail. A well known example of a heavy tail distribution used in finance is the Student- $t$.

The estimation of the tail of a distribution using EVT is often done using a method called Peaks-Over-Threshold (POT); again see Embrechts et al. (1997). The method uses a result, due to Pickands (1975), on the exceedances of $X$ calculated over a high threshold $u$.

Consider the random variable $Y=X-u$. The excess distribution function of $X$ is given by

$$
F_{u}(y)=P(X-u \leq y \mid X>u)=P(Y \leq y \mid X>u), \quad y \geq 0 .
$$

When $u$ goes to the right end point $x_{F}$ of the distribution $F_{X}{ }^{1}$, the limit distribution of the scaled exceedances is given by

$$
G_{\xi, \beta}(x)=\left\{\begin{array}{llc}
1-\left(1+\frac{\xi y}{\beta}\right)^{-1 / \xi} & \text { if } & \xi \neq 0, \\
1-e^{-y / \beta} & \text { if } & \xi=0
\end{array}\right.
$$

where

$$
\begin{array}{ccc}
x \geq 0 & \text { if } \quad \xi \geq 0, \\
0 \leq x \leq-1 / \xi \quad \text { if } \quad \xi<0,
\end{array}
$$

and $\beta>0$ is a scale parameter. This distribution is the Generalized Pareto Distribution (GPD).

From Equation (10) we have that

$$
\bar{F}(u+y)=\bar{F}(u) \bar{F}_{u}(y),
$$

where $y>0, u$ is the threshold and $\bar{F}(\cdot)$ denotes the survival function $1-F(\cdot)$. A

\footnotetext{
${ }^{1} x_{F}$ can be $+\infty$.
} 
natural estimator for $\bar{F}(u)$ is the empirical distribution function

$$
\bar{F}_{T}(u)=\frac{1}{T} \sum_{i=1}^{T} I_{X_{i}>u}=\frac{N_{u}}{T}
$$

where $N_{u}$ is the number of observations larger than $u$. For a sufficiently high threshold $u, F_{u}(y)$ can be approximated by the GPD: $\bar{F}_{u}(y) \approx 1-G_{\xi, \beta_{u}}(y)$. As a consequence the estimator for the tail distribution $\bar{F}(u+y)$ for $y>0$ is given by

$$
\widehat{F(u+y)}=\frac{N_{u}}{T}\left(1+\hat{\xi} \frac{y}{\hat{\beta}}\right)^{-1 / \hat{\xi}} .
$$

Finally, the POT method estimator for the quantile of probability $\alpha$ is given by

$$
\hat{Q}_{\alpha}(X)=u+\frac{\hat{\beta}}{\hat{\xi}}\left(\left(\frac{T}{N_{u}}(1-\alpha)\right)^{-\hat{\xi}}-1\right) .
$$

The number of exceedances $N_{u}$ is defined by the choice of threshold $u$. If $u$ is chosen too high the estimates will have a large variance and if $u$ is too small the estimates will be biased. We set a threshold $u$ such that the number of exceedances is equal to $15 \%$ of the sample data. This is a common value used in the literature; for a recent application see Chavez-Demoulin et al. (2011) and references therein.

EVT used as an unconditional method of estimating VaR suffers from the problem that EVT results described in this section assume that $X_{1}, X_{2}, \ldots, X_{T}$ are iid. In general, returns cluster and are not iid. There are generalizations of the EVT results to the non iid case (see Section 8.1 in Embrechts et al. (1997)). But the estimation of VaR by pre filtering the data should solve this problem. If the variance equation is well specified, fitting a GARCH type model assuming Normal residuals should produce consistent estimates and iid residuals even if the innovations are not normally distributed; see Bollerslev and Wooldridge (1992). This so-called Quasi-Maximum Likelihood GARCH 
coupled with an EVT model for the residuals was first implemented by McNeil and Frey (2000). In practice the variance model will never be completely well specified and a fat-tailed, as a Student- $t$ or even an asymmetric distribution for the residuals, may perform better in terms of out-of-sample VaR forecasts.

After filtering the data we apply the POT method to estimate the quantile of the standardized residuals $z_{t}=\left(r_{t}-\mu_{t}\right) / \sigma_{t}$. The conditional VaR estimate is then obtained by replacing the POT quantile in equation (8).

\section{Testing the fit of VaR models}

Using the methods described in the previous section applied to a rolling window of observations of size $w$ we obtain sequences of out-of-sample VaR estimates $\left\{\widehat{\operatorname{VaR}}_{t}^{\alpha}\right\}_{t=\omega+1, \ldots, T}$. Next we test the quality of these VaR forecasts. We have to compare the ex-ante VaR forecasts with the ex-post realized returns. This exercise is called backtesting and we follow here the procedure as in Christoffersen (1998).

Assume that we are using daily returns. Given 100 $\alpha \%$ VaR forecasts we would expect to observe 100 $(1-\alpha)$ return losses larger than the forecasted VaR every 100 days. The losses larger than the forecasted VaR are called violations.

Consider a sequence of past VaR forecasts $\left\{\widehat{\operatorname{VaR}}_{t}^{\alpha}\right\}_{t=\omega+1, \ldots, T}$ and a sequence of realized returns $\left\{r_{t}\right\}_{t=\omega+1, \ldots, T}$. In order to implement Christoffersen's backtesting procedure we start by defining the hit sequence $\left\{I_{t}\right\}_{t=1+\omega, \ldots, T}$ of VaR violations where

$$
I_{t}=\left\{\begin{array}{ccc}
1 & \text { if } & r_{t}<-\mathrm{VaR}_{t}, \\
0 & \text { if } & r_{t} \geq-\mathrm{VaR}_{t} .
\end{array}\right.
$$


We expect VaR violations to be independent and uniformly spread through time. The implication is that accordingly $I_{t}$ will have a Bernoulli distribution with parameter $1-\alpha$, where $\alpha$ usually varies between $95 \%$ and $99 \%$ depending on the confidence level required for the VaR.

\subsection{Testing the unconditional coverage}

The simplest test checks if the percentage of violations is significantly different from the corresponding VaR level $1-\alpha$. This procedure is called the unconditional coverage test.

Denote the number of zeros in the hit sequence by $T_{0}$, and the number of ones by $T_{1}$. We call $\hat{\pi}$ the observed ratio of violations, $\hat{\pi}=T_{1} / T$. A likelihood ratio test is given by

$$
\mathrm{LR}_{\mathrm{uc}}=-2 \ln [L(1-\alpha) / L(\hat{\pi})]
$$

where $L(\cdot)$ is the likelihood function of an iid Bernoulli sequence. Replacing with the appropriate function we obtain the expression for the likelihood ratio test

$$
\mathrm{LR}_{\mathrm{uc}}=-2 \ln \left[\alpha^{T_{0}}(1-\alpha)^{T_{1}} /\left\{\left(1-T_{1} / T\right)^{T_{0}}\left(T_{1} / T\right)^{T_{1}}\right\}\right] \stackrel{a}{\sim} \chi_{1}^{2},
$$

which asymptotically has a chi-square distribution with one degree of freedom.

\subsection{Testing the independence of the violations}

The unconditional coverage test checks if the number of violations is what we would expect given a level $\alpha$ for the VaR. But given that financial returns often show volatility clusters, VaR violations are also likely to cluster over time. This is an important fact. 
It is more dangerous for the financial stability of a bank to have ten VaR violations in two weeks than to have the same ten violations occurring over a period of one year. Hence, it is relevant to test the hypothesis of independence of the VaR violations. From a global point of view this question is even more important. Clusters of volatility are usually associated with events that affect several financial institutions at the same time. As a consequence these clusters of VaR violations are likely to occur simultaneously across different financial institutions. This poses a problem to the regulators with respect to systemic risk.

The Christoffersen (1998) test of independence for VaR violations assumes, under the hypothesis of dependence, that the hit sequence can be described by a first-order Markov process with transition probability matrix

$$
\Pi_{1}=\left[\begin{array}{ll}
1-\pi_{01} & \pi_{01} \\
1-\pi_{11} & \pi_{11}
\end{array}\right],
$$

where $\pi_{11}$ is the probability that tomorrow's return is a violation given that today is a violation, and $\pi_{01}$ is the probability that tomorrow's return is a violation given that today is not a violation.

Given a sample of size $T$ the likelihood function of the first-order Markov process is

$$
L\left(\Pi_{1}\right)=\left(1-\pi_{01}\right)^{T_{00}} \pi_{01}^{T_{01}}\left(1-\pi_{11}\right)^{T_{10}} \pi_{11}^{T_{11}},
$$

where $T_{i j}, i, j=0,1$, is the number of observations in the hit sequence with a $j$ following an $i$. The maximum likelihood estimates of the transition probabilities are

$$
\hat{\pi}_{01}=\frac{T_{01}}{T_{00}+T_{01}} \quad \text { and } \quad \hat{\pi}_{11}=\frac{T_{11}}{T_{10}+T_{11}} .
$$


Given a sample of returns it can happen that $T_{11}=0$. In this case the likelihood function is simply

$$
L\left(\hat{\Pi}_{1}\right)=\left(1-\hat{\pi}_{01}\right)^{T_{00}} \hat{\pi}_{01}^{T_{01}}
$$

Under the null hypothesis of independence $\pi_{01}=\pi_{11}=\pi$ and the transition matrix is

$$
\hat{\Pi}=\left[\begin{array}{cc}
1-\hat{\pi} & \hat{\pi} \\
1-\hat{\pi} & \hat{\pi}
\end{array}\right],
$$

where $\hat{\pi}=T_{1} / T$ is the estimator for the ratio of violations as in the unconditional coverage test. The likelihood function in the case of independence is then given by

$$
L(\hat{\Pi})=(1-\hat{\pi})^{T_{00}+T_{10}} \hat{\pi}^{T_{01}+T_{11}} .
$$

The likelihood ratio

$$
\mathrm{LR}_{\mathrm{ind}}=-2 \ln \left[L(\hat{\Pi}) / L\left(\hat{\Pi}_{1}\right)\right] \stackrel{a}{\sim} \chi_{1}^{2}
$$

can be used to test the independence hypothesis that $\pi_{01}=\pi_{11}$. The likelihood ratio has an asymptotic chi-square distribution with one degree of freedom.

\subsection{Testing the conditional coverage}

Christoffersen (1998) tests simultaneously if the number of violations is correct and if the VaR violations are independent. This means testing if $\pi_{01}=\pi_{11}=1-\alpha$. Christoffersen uses the likelihood ratio test

$$
\mathrm{LR}_{\mathrm{cc}}=-2 \ln \left[L(1-\alpha) / L\left(\hat{\Pi}_{1}\right)\right] \stackrel{a}{\sim} \chi_{2}^{2}
$$

which has an asymptotic chi-square distribution with two degrees of freedom. $L(1-\alpha)$ is the same as the likelihood used in equation (15). In practice it is worth noting that

$$
\mathrm{LR}_{\mathrm{cc}}=\mathrm{LR}_{\mathrm{uc}}+\mathrm{LR}_{\mathrm{ind}}
$$


the conditional coverage likelihood ratio test is the sum of the two likelihood ratios from the unconditional coverage and independence tests.

\subsection{The three color scheme from Basel II}

In practice $\mathrm{VaR}$ models are classified by the regulators according to a three color scheme devised by the Basel Committee on Banking Supervision (1996). According to this criteria a VaR model is acceptable if it falls in the "green zone", it is disputable if it falls in the "yellow zone" and it is seriously flawed if it belongs to the "red zone".

The color classification of a VaR model is a function of its number of violations. A model is in the green zone if the number of violations of the $99 \% \mathrm{VaR}$ is below the 95\% quantile of a binomial distribution with probability of success 0.01 . The model is in the yellow zone if the number of violations is between the $95 \%$ and the $99.99 \%$ quantiles of the same binomial distribution. A model is classified in the red zone if the number of violations is above the $99.99 \%$ quantile.

We add this classification to the backtesting procedure in our study in order to know how the models perform to the eyes of the regulators.

According to the colors criteria from the regulator point of view the smaller the number of violations the better the model is. That is not necessarily the case from the financial institution point of view. A number of violations much smaller than the expected number $\alpha T$ means that the VaR is being overestimated, the regulatory capital is overestimated and there is an economic cost from the loss of investment opportunity on the excessive regulatory capital. 


\section{Market capitalization in the estimation of $\mathrm{VaR}$}

We show the importance of stock market capitalization in the estimation of VaR using ten equity portfolios ranging from small to large size stocks.

We use data from the NYSE, AMEX, and NASDAQ stocks obtained from the Kenneth R. French Data Library. ${ }^{2}$ The data is originally obtained from the CRSP database consisting on stock prices. We use ten portfolios each one corresponding to a decile of the market capitalization distribution. The first decile portfolio contains the $10 \%$ of stocks with the smallest market capitalization. The second decile portfolio contains the stocks with market capitalization between the $10 \%$ and $20 \%$ decile. The tenth portfolio contains the $10 \%$ of stocks with the largest market capitalization in the market. These are equally weighted portfolios.

We opted for using these data because first, coming from large data bases it is representative of the market. Second, being a standardized database gives reliability to our conclusions and facilitates an easy replication of the results.

The data covers the period July 1, 1963 to June 30, 2012. It has been shown that during crisis periods the distributional characteristics of equity returns changes, most notably volatility increases. Hence the effect of market capitalization on VaR estimation might differ between crisis periods and non-crisis periods. We use the recession periods (see Appendix A) published by the "NBER Business Cycle Dating Committee" ${ }^{3}$ as crisis periods. Evidence for the link between market volatility and economic recessions is reported in Schwert (1989). At a firm level, Bloom et al. (2009) finds that

\footnotetext{
${ }^{2}$ Available from http://mba.tuck.dartmouth.edu/pages/faculty/ken.french.

${ }^{3}$ http://www.nber.org/cycles/cyclesmain.html
} 
there is a clear effect of recession on volatility.

We compute the VaR of daily logarithmic returns for the ten different market capitalization portfolios and for the equally weighted portfolio with all the stocks. We use different VaR methods and consider separately crises periods, non-crisis periods and the complete time period.

\subsection{Empirical results}

Table 1 presents summary statistics of the daily logarithmic returns for each portfolio. The average return decreases with market capitalization. All the portfolios present asymmetry with a considerable negative sample skewness. The kurtosis is also large. These two statistics suggest that normality would not be a reasonable assumption for the distribution of the returns.

Given a time series of daily returns for each of the portfolios considered we compute the one day ahead (out-of-sample) VaR estimates using a rolling window of past returns. We consider three different possible window lengths: $\omega=250, \omega=500$, and $\omega=1000$. We include in this paper the numerical results for $\omega=1000$. The tables with the results for $\omega=250$ and $\omega=500$ are available from the author on request. The conclusions are consistent across the three window lengths. For each portfolio and window length we obtain a sequence of VaR estimates that can be backtested against the observed returns.

In this study we consider crisis periods, non-crisis periods and the full sample. The samples for the three different cases naturally have different sizes. In order to keep the comparability of the backtesting for different samples we backtest all the models using 
Table 1: Summary statistics of the returns on the different market size portfolios.

\begin{tabular}{lccccrrc} 
Portfolio/Cap & Mean & Std. Dev. & Skewness & Kurtosis & Min & Max & Semi Std.Dev \\
\hline Lower Decile & 0.1054 & 0.7480 & -0.7580 & 11.875 & -8.44 & 7.19 & 0.7666 \\
2nd Decile & 0.0567 & 1.1049 & -0.2884 & 13.008 & -12.60 & 10.03 & 1.1526 \\
3rd Decile & 0.0570 & 1.0996 & -0.3474 & 10.517 & -12.21 & 9.21 & 1.1410 \\
4th Decile & 0.0530 & 1.0900 & -0.3297 & 10.908 & -12.72 & 9.61 & 1.1281 \\
5th Decile & 0.0536 & 1.0922 & -0.3177 & 10.943 & -12.29 & 10.42 & 1.1245 \\
6th Decile & 0.0512 & 1.0366 & -0.3559 & 11.819 & -13.53 & 11.23 & 1.0621 \\
7th Decile & 0.0510 & 1.0464 & -0.3493 & 14.615 & -14.53 & 12.94 & 1.0692 \\
8th Decile & 0.0489 & 1.0565 & -0.2888 & 15.133 & -15.35 & 13.64 & 1.0751 \\
9th Decile & 0.0475 & 1.0476 & -0.4158 & 18.858 & -18.00 & 13.43 & 1.0611 \\
Higher Decile & 0.0426 & 1.0731 & -0.3517 & 20.097 & -19.52 & 14.20 & 1.0681 \\
All stocks & 0.0567 & 0.9932 & -0.4308 & 13.607 & -13.64 & 11.12 & 1.0317 \\
\hline
\end{tabular}

Notes: Summary statistics of the daily returns on the ten portfolios from small to large market capitalization stocks and for the portfolio with all the stocks. The data covers the period from July 1, 1963 to June 30, 2012. In the last column Semi Std.Dev stands for the square root of the negative semi-variance. The negative semi-variance measures the dispersion of all observations falling below the mean and it is defined as $\left(\sigma^{2}\right)^{-}=E\left([E(R)-R]^{+}\right)^{2}$.

series of VaR estimates with the same length. As the crisis periods listed in Appendix A cover 3081 days of the total sample period we use the last 3081 daily VaR estimates in all the backtesting performed.

If the $100 \alpha \%$ VaR is well estimated then, on average, $100(1-\alpha) \%$ of the returns will produce a VaR violation. In this study we use the level of $99 \%$ for the VaR. We choose to use this level of confidence because the directives of the Basel Committee on Banking Supervision (1996) set the required regulatory capital as a function of the performance of $99 \%$ VaR internal models.

The most popular VaR estimation method in industry is the simple unconditional historical simulation. Table 2 presents the results from estimating the $99 \%$ VaR for the different portfolios and corresponding backtesting using a window of $\omega=1000$ observations. $\overline{\mathrm{VaR}}$ denotes the average VaR. The percentage number of violations which fall in the green, yellow and red Basel II color classification have normal, italic and bold 
Table 2: Performance of unconditional historical simulation VaR method

\begin{tabular}{|c|c|c|c|c|c|c|c|c|c|c|c|}
\hline Decile & Lower & 2nd & $3 \mathrm{rd}$ & 4 th & 5 th & 6 th & 7 th & 8 th & 9th & Higher & All \\
\hline \multicolumn{12}{|c|}{ Non-crisis periods } \\
\hline$\overline{\overline{\mathrm{VaR}}}$ & 2.07 & 3.45 & 3.43 & 3.32 & 3.42 & 3.15 & 3.36 & 3.28 & 3.19 & 3.16 & 3.07 \\
\hline$\%$ Viol. & 0.77 & 0.64 & 0.55 & 0.61 & 0.68 & 0.77 & 0.74 & 0.84 & 0.90 & 1.00 & 0.74 \\
\hline $\mathrm{LR}_{\mathrm{uc}}$ & 0.20 & 0.03 & 0.00 & 0.02 & 0.05 & 0.20 & 0.13 & 0.37 & 0.60 & 0.96 & 0.13 \\
\hline $\mathrm{LR}_{\text {ind }}$ & 0.00 & 0.11 & 0.65 & 0.61 & 0.58 & 0.53 & 0.16 & 0.21 & 0.02 & 0.31 & 0.54 \\
\hline $\mathrm{LR}_{\mathrm{cc}}$ & 0.00 & 0.03 & 0.02 & 0.06 & 0.14 & 0.36 & 0.12 & 0.31 & 0.07 & 0.60 & 0.28 \\
\hline \multicolumn{12}{|c|}{ Crisis periods } \\
\hline$\overline{\mathrm{VaR}}$ & 2.28 & 2.99 & 3.05 & 2.99 & 3.07 & 3.04 & 2.92 & 2.81 & 2.67 & 2.83 & 2.80 \\
\hline$\%$ Viol. & 1.56 & 2.04 & 1.98 & 2.24 & 2.50 & 2.34 & 2.50 & 2.60 & 2.47 & 2.76 & 2.34 \\
\hline $\mathrm{LR}_{\mathrm{uc}}$ & 0.00 & 0.00 & 0.00 & 0.00 & 0.00 & 0.00 & 0.00 & 0.00 & 0.00 & 0.00 & 0.00 \\
\hline $\mathrm{LR}_{\text {ind }}$ & 0.00 & 0.00 & 0.00 & 0.00 & 0.00 & 0.00 & 0.00 & 0.00 & 0.04 & 0.00 & 0.00 \\
\hline $\mathrm{LR}_{\mathrm{cc}}$ & 0.00 & 0.00 & 0.00 & 0.00 & 0.00 & 0.00 & 0.00 & 0.00 & 0.00 & 0.00 & 0.00 \\
\hline \multicolumn{12}{|c|}{ Full sample period } \\
\hline$\overline{\mathrm{VaR}}$ & 2.52 & 4.03 & 4.08 & 3.97 & 4.14 & 3.87 & 4.03 & 3.91 & 3.84 & 3.89 & 3.70 \\
\hline$\%$ Viol. & 1.26 & 1.39 & 1.39 & 1.46 & 1.55 & 1.65 & 1.72 & 1.81 & 1.68 & 1.68 & 1.55 \\
\hline $\mathrm{LR}_{\mathrm{uc}}$ & 0.15 & 0.03 & 0.01 & 0.01 & 0.00 & 0.00 & 0.00 & 0.00 & 0.00 & 0.00 & 0.00 \\
\hline $\mathrm{LR}_{\text {ind }}$ & 0.00 & 0.14 & 0.13 & 0.00 & 0.00 & 0.06 & 0.00 & 0.00 & 0.01 & 0.06 & 0.04 \\
\hline $\mathrm{LR}_{\mathrm{cc}}$ & 0.00 & 0.03 & 0.03 & 0.00 & 0.00 & 0.00 & 0.00 & 0.00 & 0.00 & 0.00 & 0.00 \\
\hline
\end{tabular}

Notes: The table reports the results from using the historical simulation method to estimate the $99 \%$ VaR across the different deciles of market capitalization using a rolling window of 1000 days. $\overline{\mathrm{VaR}}$ denotes the average VaR. The percentage number of violations which fall in the green, yellow and red Basel II color classification have normal, italic and bold type face respectively. $\mathrm{LR}_{\mathrm{uc}}$ are the p-values obtained from the unconditional coverage test in equation (16). $\mathrm{LR}_{\text {ind }}$ and $\mathrm{LR}_{\mathrm{cc}}$ are the p-values of the independence test (see equation (22)) and conditional coverage test (see equation (24)) respectively. 
type face respectively. $\mathrm{LR}_{\mathrm{uc}}$ are the p-values obtained from the unconditional coverage test given in equation (16). $\mathrm{LR}_{\text {ind }}$ and $\mathrm{LR}_{\mathrm{cc}}$ are the p-values of the independence test (see equation (22)) and conditional coverage test (see equation (24)) respectively.

From Table 2 we observe that the percentage of violations observed for the full period sample is higher than the target level of $1 \%$ chosen for the VaR. In general the excess percentage of violations over the $1 \%$ level increases with the market capitalization. As a consequence the historical simulation method falls in the yellow zone only in the five lower decile portfolios. For all the higher decile portfolios the method is in the red zone.

This result is actually an averaging of the performance for non-crisis and crisis periods. In the same table we observe that the VaR is overestimated for the non-crisis periods and is underestimated during the crisis periods.

Although the Basel classification is green for the percentage violation during the non-crisis periods the economic capital is actually being overestimated in relation to what would be necessary to maintain a level of $99 \%$ VaR with the consequent investment opportunity cost. During the crisis periods the VaR is underestimated and the risk is higher. The regulatory capital is insufficient to overcome possible losses with a 99\% VaR confidence level. Hence, by backtesting over the full sample period we would conclude that the historical simulation VaR model performs better than it actually does. In fact, whether during crises or non-crisis periods, the model always performs poorly.

From Table 2 we can also observe that the difference between the observed percentage of violations and the target VaR level increases with the market capitalization 
Table 3: Performance of unconditional Normal VaR estimates

\begin{tabular}{|c|c|c|c|c|c|c|c|c|c|c|c|}
\hline Decile & Lower & 2nd & $3 r d$ & 4th & 5 th & 6th & 7 th & 8th & 9th & Higher & All \\
\hline \multicolumn{12}{|c|}{ Non-crisis periods } \\
\hline$\overline{\mathrm{VaR}}$ & 1.51 & 2.84 & 2.85 & 2.81 & 2.85 & 2.60 & 2.64 & 2.70 & 2.64 & 2.61 & 2.49 \\
\hline$\%$ Viol. & 1.94 & 1.52 & 1.49 & 1.46 & 1.29 & 1.59 & 1.49 & 1.52 & 1.26 & 1.59 & 1.59 \\
\hline $\mathrm{LR}_{\mathrm{uc}}$ & 0.00 & 0.00 & 0.00 & 0.01 & 0.11 & 0.00 & 0.01 & 0.00 & 0.15 & 0.00 & 0.00 \\
\hline $\mathrm{LR}_{\text {ind }}$ & 0.00 & 0.00 & 0.01 & 0.03 & 0.00 & 0.00 & 0.00 & 0.03 & 0.01 & 0.23 & 0.00 \\
\hline $\mathrm{LR}_{\mathrm{cc}}$ & 0.00 & 0.00 & 0.00 & 0.00 & 0.00 & 0.00 & 0.00 & 0.00 & 0.01 & 0.00 & 0.00 \\
\hline \multicolumn{12}{|c|}{ Crisis periods } \\
\hline$\overline{\mathrm{VaR}}$ & 1.64 & 2.26 & 2.36 & 2.39 & 2.43 & 2.36 & 2.30 & 2.34 & 2.32 & 2.44 & 2.17 \\
\hline$\%$ Viol. & 4.05 & 4.31 & 4.44 & 4.41 & 4.44 & 4.21 & 4.93 & 4.41 & 4.64 & 4.57 & 4.41 \\
\hline $\mathrm{LR}_{\mathrm{uc}}$ & 0.00 & 0.00 & 0.00 & 0.00 & 0.00 & 0.00 & 0.00 & 0.00 & 0.00 & 0.00 & 0.00 \\
\hline $\mathrm{LR}_{\text {ind }}$ & 0.00 & 0.00 & 0.00 & 0.00 & 0.00 & 0.00 & 0.00 & 0.00 & 0.00 & 0.00 & 0.00 \\
\hline $\mathrm{LR}_{\mathrm{cc}}$ & 0.00 & 0.00 & 0.00 & 0.00 & 0.00 & 0.00 & 0.00 & 0.00 & 0.00 & 0.00 & 0.00 \\
\hline \multicolumn{12}{|c|}{ Full sample period } \\
\hline$\overline{\mathrm{VaR}}$ & 1.87 & 3.40 & 3.46 & 3.46 & 3.54 & 3.23 & 3.26 & 3.31 & 3.24 & 3.22 & 3.07 \\
\hline \% Viol. & 2.95 & 2.79 & 2.72 & 2.75 & 2.46 & 2.72 & 3.08 & 2.79 & 2.62 & 2.59 & 2.75 \\
\hline $\mathrm{LR}_{\mathrm{uc}}$ & 0.00 & 0.00 & 0.00 & 0.00 & 0.00 & 0.00 & 0.00 & 0.00 & 0.00 & 0.00 & 0.00 \\
\hline $\mathrm{LR}_{\text {ind }}$ & 0.00 & 0.00 & 0.00 & 0.00 & 0.01 & 0.00 & 0.00 & 0.00 & 0.00 & 0.02 & 0.11 \\
\hline $\mathrm{LR}_{\mathrm{cc}}$ & 0.00 & 0.00 & 0.00 & 0.00 & 0.00 & 0.00 & 0.00 & 0.00 & 0.00 & 0.00 & 0.00 \\
\hline
\end{tabular}

Notes: Results from using the unconditional Normal method to estimate the $99 \%$ VaR across the different deciles of market capitalization using a rolling window of 1000 days. See also notes in Table 2 .

during crises and decreases during non-crisis periods. This indicates that accurate unconditional VaR estimates are more difficult to obtain for different market capitalization in different states of the market.

To further investigate the differences between VaR estimates for different market capitalization sizes we use three unconditional fully parametric methods as in equation (5). We use the unconditional method with a Normal distribution which should be more appropriate for the non-crisis periods, with the Student- $t$ distribution which should work better during crisis periods, and with EVT which allows for asymmetry of the returns distribution.

The results for the unconditional Normal method are reported in Table 3. The quality of the VaR estimates decreases for both crisis and non-crisis periods. The VaR is underestimated for all the deciles. There is again a large difference in the observed 
Table 4: Performance of unconditional Student- $t$ VaR estimates

\begin{tabular}{|c|c|c|c|c|c|c|c|c|c|c|c|}
\hline Decile & Lower & 2nd & $3 r d$ & 4th & 5 th & 6th & 7 th & 8th & 9th & Higher & All \\
\hline \multicolumn{12}{|c|}{ Non-crisis periods } \\
\hline$\overline{\mathrm{VaR}}$ & 2.18 & 3.48 & 3.45 & 3.39 & 3.48 & 3.25 & 3.37 & 3.45 & 3.43 & 3.43 & 3.17 \\
\hline$\%$ Viol. & 0.61 & 0.71 & 0.68 & 0.61 & 0.74 & 0.97 & 0.81 & 0.87 & 0.87 & 0.90 & 0.81 \\
\hline $\mathrm{LR}_{\mathrm{uc}}$ & 0.02 & 0.09 & 0.05 & 0.02 & 0.13 & 0.88 & 0.27 & 0.48 & 0.48 & 0.60 & 0.27 \\
\hline $\mathrm{LR}_{\text {ind }}$ & 0.61 & 0.14 & 0.00 & 0.61 & 0.16 & 0.43 & 0.19 & 0.23 & 0.23 & 0.25 & 0.51 \\
\hline $\mathrm{LR}_{\mathrm{cc}}$ & 0.06 & 0.08 & 0.00 & 0.06 & 0.12 & 0.72 & 0.24 & 0.38 & 0.38 & 0.45 & 0.44 \\
\hline \multicolumn{12}{|c|}{ Crisis periods } \\
\hline$\overline{\mathrm{VaR}}$ & 2.24 & 2.83 & 2.91 & 2.91 & 2.98 & 2.89 & 2.83 & 2.86 & 2.79 & 2.93 & 2.70 \\
\hline$\%$ Viol. & 2.20 & 2.53 & 2.30 & 2.36 & 2.85 & 2.82 & 2.85 & 2.85 & 2.72 & 2.85 & 2.46 \\
\hline $\mathrm{LR}_{\mathrm{uc}}$ & 0.00 & 0.00 & 0.00 & 0.00 & 0.00 & 0.00 & 0.00 & 0.00 & 0.00 & 0.00 & 0.00 \\
\hline $\mathrm{LR}_{\text {ind }}$ & 0.00 & 0.00 & 0.02 & 0.00 & 0.00 & 0.00 & 0.00 & 0.00 & 0.00 & 0.00 & 0.00 \\
\hline $\mathrm{LR}_{\mathrm{cc}}$ & 0.00 & 0.00 & 0.00 & 0.00 & 0.00 & 0.00 & 0.00 & 0.00 & 0.00 & 0.00 & 0.00 \\
\hline \multicolumn{12}{|c|}{ Full sample period } \\
\hline$\overline{\mathrm{VaR}}$ & 2.53 & 4.09 & 4.11 & 4.08 & 4.24 & 3.99 & 4.12 & 4.18 & 4.15 & 4.16 & 3.82 \\
\hline$\%$ Viol. & 1.65 & 1.72 & 1.49 & 1.42 & 1.62 & 1.75 & 1.78 & 1.88 & 1.72 & 1.75 & 1.55 \\
\hline $\mathrm{LR}_{\mathrm{uc}}$ & 0.00 & 0.00 & 0.01 & 0.02 & 0.00 & 0.00 & 0.00 & 0.00 & 0.00 & 0.00 & 0.00 \\
\hline $\mathrm{LR}_{\text {ind }}$ & 0.00 & 0.07 & 0.18 & 0.02 & 0.00 & 0.08 & 0.00 & 0.02 & 0.01 & 0.08 & 0.04 \\
\hline $\mathrm{LR}_{\mathrm{cc}}$ & 0.00 & 0.00 & 0.01 & 0.00 & 0.00 & 0.00 & 0.00 & 0.00 & 0.00 & 0.00 & 0.00 \\
\hline
\end{tabular}

Notes: Results from using the unconditional Student- $t$ method to estimate the $99 \%$ VaR across the different deciles of market capitalization using a rolling window of 1000 days. See also notes in Table 2.

percentage violations during crises and non-crisis periods. Similarly to the historical simulation method the difference between the observed percentage of violations and the target VaR level increases with market capitalization during crises and decreases during non-crises.

Given the high sample kurtosis of the returns reported in Table 1 we backtest the unconditional Student- $t$ method. The results are listed in Table 4. The Student- $t$ still underestimates VaR during crisis periods. There is a large difference between the overestimation of $\mathrm{VaR}$ during non-crisis periods and the underestimation during crisis periods.

Despite the problem with the lack of independence of the returns pointed out in Section 2.3, we implemented the unconditional EVT method. The returns reveal asymmetry hence using EVT should overcome the symmetry of the Normal and Student- $t$. 
Table 5: Performance of unconditional EVT VaR estimates

\begin{tabular}{|c|c|c|c|c|c|c|c|c|c|c|c|}
\hline Decile & Lower & 2nd & $3 \mathrm{rd}$ & 4 th & 5 th & 6 th & 7 th & 8 th & 9 th & Higher & All \\
\hline \multicolumn{12}{|c|}{ Non-crisis periods } \\
\hline$\overline{\mathrm{VaR}}$ & 1.94 & 3.38 & 3.35 & 3.26 & 3.34 & 3.10 & 3.18 & 3.23 & 3.15 & 3.12 & 2.99 \\
\hline$\%$ Viol. & 0.94 & 0.68 & 0.58 & 0.68 & 0.77 & 0.84 & 0.81 & 0.87 & 0.90 & 1.00 & 0.87 \\
\hline $\mathrm{LR}_{\mathrm{uc}}$ & 0.74 & 0.05 & 0.01 & 0.05 & 0.19 & 0.37 & 0.27 & 0.48 & 0.60 & 0.97 & 0.48 \\
\hline $\mathrm{LR}_{\text {ind }}$ & 0.00 & 0.13 & 0.63 & 0.58 & 0.17 & 0.49 & 0.51 & 0.23 & 0.25 & 0.03 & 0.48 \\
\hline $\mathrm{LR}_{\mathrm{cc}}$ & 0.00 & 0.05 & 0.03 & 0.14 & 0.17 & 0.53 & 0.44 & 0.38 & 0.45 & 0.11 & 0.60 \\
\hline \multicolumn{12}{|c|}{ Crisis periods } \\
\hline$\overline{\mathrm{VaR}}$ & 2.24 & 2.93 & 2.96 & 2.94 & $2.98^{1}$ & 2.88 & 2.82 & 2.81 & 2.73 & 2.81 & 2.70 \\
\hline$\%$ Viol. & 2.01 & 2.33 & 2.33 & 2.36 & 2.66 & 2.66 & 2.92 & 2.79 & 2.62 & 2.98 & 2.46 \\
\hline $\mathrm{LR}_{\mathrm{uc}}$ & 0.00 & 0.00 & 0.00 & 0.00 & 0.00 & 0.00 & 0.00 & 0.00 & 0.00 & 0.00 & 0.00 \\
\hline $\mathrm{LR}_{\text {ind }}$ & 0.00 & 0.00 & 0.03 & 0.00 & 0.00 & 0.00 & 0.00 & 0.00 & 0.00 & 0.00 & 0.00 \\
\hline $\mathrm{LR}_{\mathrm{cc}}$ & 0.00 & 0.00 & 0.00 & 0.00 & 0.00 & 0.00 & 0.00 & 0.00 & 0.00 & 0.00 & 0.00 \\
\hline \multicolumn{12}{|c|}{ Full sample period } \\
\hline$\overline{\mathrm{VaR}}$ & 2.40 & 3.99 & 3.98 & 3.91 & 4.04 & 3.76 & 3.87 & 3.90 & 3.84 & 3.81 & 3.60 \\
\hline$\%$ Viol. & 1.59 & 1.72 & 1.59 & 1.62 & 1.72 & 1.75 & 1.88 & 1.88 & 1.81 & 1.78 & 1.72 \\
\hline $\mathrm{LR}_{\mathrm{uc}}$ & 0.00 & 0.00 & 0.00 & 0.00 & 0.00 & 0.00 & 0.00 & 0.00 & 0.00 & 0.00 & 0.00 \\
\hline $\mathrm{LR}_{\text {ind }}$ & 0.00 & 0.07 & 0.23 & 0.00 & 0.01 & 0.08 & 0.00 & 0.02 & 0.02 & 0.09 & 0.07 \\
\hline $\mathrm{LR}_{\mathrm{cc}}$ & 0.00 & 0.00 & 0.00 & 0.00 & 0.00 & 0.00 & 0.00 & 0.00 & 0.00 & 0.00 & 0.00 \\
\hline
\end{tabular}

Notes: Results from using the unconditional EVT method to estimate the $99 \%$ VaR across the different deciles of market capitalization using a rolling window of 1000 days. See also notes in Table 2.

The results, reported in Table 5, do not point to different conclusions than the unconditional Student- $t$ method.

If we take the perspective of which method performs best in which situation then, using a window of $\omega=1000$, during non-crisis periods the best performing method is the unconditional EVT. During crisis periods and using the full sample the best performing method is the unconditional historical simulation. This result might be surprising as we would expect that the EVT method would outperform during crisis periods where extreme losses occur more frequently. For a window of $\omega=250$ the historical simulation method performs the best in any case. For $\omega=500$, during crisis, non-crisis or using the full sample, historical simulation is the best performing model for smaller market capitalization stocks, and Student- $t$ is the best model for larger market capitalization stocks. 


\subsection{Empirical results from conditional VaR methods}

In order to evaluate the effect of market capitalization on the performance of conditional methods for estimating VaR we consider the following models: filtered historical simulation (where the filters are an $\operatorname{AR}(1)-\operatorname{GARCH}(1,1)$ with Student- $t$ and Normal innovations); a simple $\operatorname{AR}(1)-\operatorname{GARCH}(1,1)$ with Student- $t$ innovations; a simple AR(1)-GARCH(1,1) with Normal innovations; filtered EVT (where the filters are an $\operatorname{AR}(1)-G A R C H(1,1)$ with Student- $t$ and Normal innovations).

To keep the readability of the paper we present in this section the results for the models with Student- $t$ innovations and window size $\omega=1000$. The results from these models are representative of the conclusions relating the importance of market capitalization in the estimation of VaR. For reference the tables with the results obtained with the models having Normal innovations and window size $\omega=1000$ are available in Appendix B. The results obtained with window sizes $\omega=250$ and $\omega=500$ are available from the author on request.

We report in Tables 6,7 and 8 the results for the conditional VaR forecasts. In general the three methods underestimate VaR. The p-values of the independence test are high for the three models. The conditional model with filtered historical simulation has high p-values for the three backtesting tests. The other two models, AR-GARCH- $t$ and AR-GARCH- $t$ filtered EVT, have low p-values for the unconditional and conditional coverage tests.

The performance for the full sample is systematically better than for the crisis and non-crisis periods separately. This is an important result with repercussions in practice. If we backtest a model using the full sample we can obtain good backtesting 
Table 6: Performance of Conditional VaR forecasts.

\begin{tabular}{|c|c|c|c|c|c|c|c|c|c|c|c|}
\hline Decile & Lower & 2nd & $3 \mathrm{rd}$ & 4 th & 5 th & 6 th & 7 th & 8th & 9 th & Higher & All \\
\hline \multicolumn{12}{|c|}{ Non-crisis periods } \\
\hline$\overline{\mathrm{VaR}}$ & 1.37 & 2.54 & 2.54 & 2.45 & 2.40 & 2.12 & 2.08 & 2.15 & 2.07 & 2.05 & 2.09 \\
\hline$\%$ Viol. & 1.65 & 1.23 & 1.23 & 1.46 & 1.29 & 1.46 & 1.72 & 1.42 & 1.46 & 1.49 & 1.49 \\
\hline $\mathrm{LR}_{\mathrm{uc}}$ & 0.00 & 0.20 & 0.20 & 0.01 & 0.11 & 0.01 & 0.00 & 0.02 & 0.01 & 0.01 & 0.01 \\
\hline $\mathrm{LR}_{\text {ind }}$ & 0.18 & 0.32 & 0.32 & 0.24 & 0.29 & 0.66 & 0.16 & 0.63 & 0.24 & 0.23 & 0.23 \\
\hline $\mathrm{LR}_{\mathrm{cc}}$ & 0.00 & 0.27 & 0.27 & 0.02 & 0.16 & 0.05 & 0.00 & 0.07 & 0.02 & 0.01 & 0.01 \\
\hline \multicolumn{12}{|c|}{ Crisis periods } \\
\hline$\overline{\mathrm{VaR}}$ & 1.86 & 2.96 & 3.10 & 3.13 & 3.22 & 3.12 & 3.18 & 3.24 & 3.21 & 3.35 & 2.92 \\
\hline$\%$ Viol. & 1.81 & 1.59 & 1.75 & 1.49 & 1.55 & 1.59 & 1.85 & 1.65 & 1.49 & 1.20 & 1.72 \\
\hline $\mathrm{LR}_{\mathrm{uc}}$ & 0.00 & 0.00 & 0.00 & 0.01 & 0.00 & 0.00 & 0.00 & 0.00 & 0.01 & 0.27 & 0.00 \\
\hline $\mathrm{LR}_{\text {ind }}$ & 0.14 & 0.20 & 0.16 & 0.68 & 0.21 & 0.76 & 0.84 & 0.80 & 0.63 & 0.33 & 0.85 \\
\hline $\mathrm{LR}_{\mathrm{cc}}$ & 0.00 & 0.00 & 0.00 & 0.03 & 0.00 & 0.00 & 0.00 & 0.00 & 0.03 & 0.34 & 0.00 \\
\hline \multicolumn{12}{|c|}{ Full sample period } \\
\hline$\overline{\mathrm{VaR}}$ & 1.88 & 3.66 & 3.70 & 3.65 & 3.67 & 3.28 & 3.31 & 3.36 & 3.26 & 3.16 & 3.20 \\
\hline$\%$ Viol. & 1.52 & 1.20 & 1.10 & 1.00 & 1.07 & 1.26 & 1.55 & 1.13 & 1.26 & 1.23 & 1.16 \\
\hline $\mathrm{LR}_{\mathrm{uc}}$ & 0.00 & 0.27 & 0.56 & 0.97 & 0.69 & 0.15 & 0.00 & 0.45 & 0.15 & 0.20 & 0.36 \\
\hline $\mathrm{LR}_{\text {ind }}$ & 0.22 & 0.33 & 0.00 & 0.41 & 0.39 & 0.50 & 0.21 & 0.36 & 0.31 & 0.32 & 0.34 \\
\hline $\mathrm{LR}_{\mathrm{cc}}$ & 0.01 & 0.34 & 0.00 & 0.72 & 0.64 & 0.29 & 0.00 & 0.50 & 0.21 & 0.27 & 0.42 \\
\hline
\end{tabular}

Notes: Results from using the conditional AR(1)-GARCH(1,1)-t method to estimate the $99 \%$ VaR across the different deciles of market capitalization using a rolling window of 1000 days. See also notes in Table 2.

Table 7: Performance of Conditional VaR forecasts.

\begin{tabular}{|c|c|c|c|c|c|c|c|c|c|c|c|}
\hline Decile & Lower & 2nd & $3 r d$ & 4 th & 5 th & 6 th & 7 th & 8 th & 9 th & Higher & All \\
\hline \multicolumn{12}{|c|}{ Non-crisis periods } \\
\hline$\overline{\mathrm{VaR}}$ & 1.60 & 2.65 & 2.59 & 2.49 & 2.46 & 2.23 & 2.18 & 2.22 & 2.18 & 2.10 & 2.18 \\
\hline$\%$ Viol. & 1.13 & 1.10 & 0.97 & 1.23 & 1.20 & 1.20 & 1.10 & 1.36 & 1.42 & 1.29 & 1.13 \\
\hline $\mathrm{LR}_{\mathrm{uc}}$ & 0.45 & 0.56 & 0.88 & 0.20 & 0.27 & 0.27 & 0.56 & 0.05 & 0.02 & 0.11 & 0.45 \\
\hline $\mathrm{LR}_{\text {ind }}$ & 0.36 & 0.37 & 0.43 & 0.32 & 0.33 & 0.45 & 0.37 & 0.58 & 0.25 & 0.29 & 0.36 \\
\hline $\mathrm{LR}_{\mathrm{cc}}$ & 0.50 & 0.57 & 0.72 & 0.27 & 0.34 & 0.42 & 0.57 & 0.13 & 0.04 & 0.16 & 0.50 \\
\hline \multicolumn{12}{|c|}{ Crisis periods } \\
\hline$\overline{\mathrm{VaR}}$ & 2.08 & 3.22 & 3.32 & 3.38 & 3.46 & 3.31 & 3.44 & 3.38 & 3.38 & 3.39 & 3.14 \\
\hline$\%$ Viol. & 1.13 & 0.94 & 1.07 & 0.94 & 0.94 & 1.10 & 1.26 & 1.26 & 1.16 & 1.13 & 1.00 \\
\hline $\mathrm{LR}_{\mathrm{uc}}$ & 0.45 & 0.74 & 0.69 & 0.74 & 0.74 & 0.56 & 0.15 & 0.15 & 0.36 & 0.45 & 0.97 \\
\hline $\mathrm{LR}_{\text {ind }}$ & 0.36 & 0.45 & 0.39 & 0.45 & 0.45 & 0.37 & 0.31 & 0.50 & 0.34 & 0.40 & 0.41 \\
\hline $\mathrm{LR}_{\mathrm{cc}}$ & 0.50 & 0.51 & 0.64 & 0.71 & 0.71 & 0.57 & 0.21 & 0.29 & 0.42 & 0.53 & 0.72 \\
\hline \multicolumn{12}{|c|}{ Full sample period } \\
\hline$\overline{\mathrm{VaR}}$ & 2.12 & 3.77 & 3.73 & 3.63 & 3.67 & 3.34 & 3.43 & 3.39 & 3.33 & 3.14 & 3.26 \\
\hline$\%$ Viol. & 1.16 & 1.00 & 1.07 & 1.10 & 1.07 & 1.28 & 1.16 & 1.26 & 1.29 & 1.23 & 1.00 \\
\hline $\mathrm{LR}_{\mathrm{uc}}$ & 0.36 & 0.97 & 0.69 & 0.56 & 0.69 & 0.11 & 0.36 & 0.15 & 0.11 & 0.20 & 0.97 \\
\hline $\mathrm{LR}_{\text {ind }}$ & 0.34 & 0.41 & 0.00 & 0.37 & 0.39 & 0.53 & 0.34 & 0.31 & 0.29 & 0.48 & 0.41 \\
\hline $\mathrm{LR}_{\mathrm{cc}}$ & 0.42 & 0.72 & 0.00 & 0.57 & 0.64 & 0.23 & 0.42 & 0.21 & 0.16 & 0.35 & 0.72 \\
\hline
\end{tabular}

Notes: Results from using the conditional AR(1)-GARCH(1,1)- $t$ filtered historical simulation method to estimate the $99 \%$ VaR across the different deciles of market capitalization using a rolling window of 1000 days. See also notes in Table 2. 
Table 8: Performance of Conditional VaR forecasts.

\begin{tabular}{|c|c|c|c|c|c|c|c|c|c|c|c|}
\hline Decile & Lower & 2nd & $3 r d$ & 4 th & 5 th & 6 th & 7 th & 8 th & 9 th & Higher & All \\
\hline \multicolumn{12}{|c|}{ Non-crisis periods } \\
\hline$\overline{\mathrm{VaR}}$ & 1.29 & 2.52 & 2.62 & 2.49 & 2.37 & 2.05 & 2.03 & 2.11 & 1.90 & 1.86 & 2.02 \\
\hline$\%$ Viol. & 2.43 & 1.94 & 1.42 & 1.68 & 2.04 & 2.10 & 2.01 & 1.72 & 2.40 & 2.43 & 1.97 \\
\hline $\mathrm{LR}_{\mathrm{uc}}$ & 0.00 & 0.00 & 0.02 & 0.00 & 0.00 & 0.00 & 0.00 & 0.00 & 0.00 & 0.00 & 0.00 \\
\hline $\mathrm{LR}_{\text {ind }}$ & 0.39 & 0.00 & 0.02 & 0.17 & 0.53 & 0.21 & 0.50 & 0.30 & 0.37 & 0.14 & 0.48 \\
\hline $\mathrm{LR}_{\mathrm{cc}}$ & 0.00 & 0.00 & 0.00 & 0.00 & 0.00 & 0.00 & 0.00 & 0.00 & 0.00 & 0.00 & 0.00 \\
\hline \multicolumn{12}{|c|}{ Crisis periods } \\
\hline$\overline{\mathrm{VaR}}$ & 1.69 & 2.69 & 2.88 & 3.00 & 2.98 & 2.87 & 2.94 & 3.10 & 2.75 & 2.88 & 2.72 \\
\hline$\%$ Viol. & 2.49 & 2.53 & 2.43 & 2.07 & 2.33 & 2.46 & 2.33 & 2.04 & 2.85 & 2.69 & 2.33 \\
\hline $\mathrm{LR}_{\mathrm{uc}}$ & 0.00 & 0.00 & 0.00 & 0.00 & 0.00 & 0.00 & 0.00 & 0.00 & 0.00 & 0.00 & 0.00 \\
\hline $\mathrm{LR}_{\text {ind }}$ & 0.43 & 0.46 & 0.79 & 0.55 & 0.33 & 0.41 & 0.74 & 0.53 & 0.69 & 0.77 & 0.11 \\
\hline $\mathrm{LR}_{\mathrm{cc}}$ & 0.00 & 0.00 & 0.00 & 0.00 & 0.00 & 0.00 & 0.00 & 0.00 & 0.00 & 0.00 & 0.00 \\
\hline \multicolumn{12}{|c|}{ Full sample period } \\
\hline$\overline{\mathrm{VaR}}$ & 1.85 & 3.59 & 3.77 & 3.76 & 3.63 & 3.20 & 3.17 & 3.35 & 2.92 & 2.81 & 3.11 \\
\hline$\%$ Viol. & 1.49 & 1.52 & 1.03 & 0.97 & 1.39 & 1.62 & 1.68 & 1.10 & 2.10 & 2.36 & 1.36 \\
\hline $\mathrm{LR}_{\mathrm{uc}}$ & 0.01 & 0.00 & 0.83 & 0.48 & 0.03 & 0.00 & 0.00 & 0.56 & 0.00 & 0.00 & 0.05 \\
\hline $\mathrm{LR}_{\text {ind }}$ & 0.23 & 0.22 & 0.00 & 0.48 & 0.26 & 0.78 & 0.82 & 0.38 & 0.64 & 0.51 & 0.27 \\
\hline $\mathrm{LR}_{\mathrm{cc}}$ & 0.01 & 0.01 & 0.00 & 0.60 & 0.06 & 0.00 & 0.00 & 0.58 & 0.00 & 0.00 & 0.08 \\
\hline
\end{tabular}

Notes: Results from using the conditional AR(1)-GARCH(1,1)-t filtered EVT method to estimate the $99 \%$ VaR across the different deciles of market capitalization using a rolling window of 1000 days. See also notes in Table 2 .

results although the model performs poorly during crisis periods and during non-crisis periods. This means that VaR is always wrongly estimated if we don't take crisis and non-crisis periods into consideration.

In terms of best performing models, the AR-GARCH models with Normal and Student- $t$ innovations filtered historical simulation have the best performance for every period and window size.

\section{Significance of market capitalization}

In this section we want to test if the number of violations produced by a model significantly changes with the market capitalization of a portfolio.

For the several models we regress the percentage of VaR violations on the market 
Table 9: Relation between market capitalization and the percentage number of violations.

\begin{tabular}{lccc} 
VaR Model & Non-crisis periods & Crisis periods & All sample \\
\hline Unconditional historical simulation & $0.035^{* * *}$ & $0.106^{* * *}$ & $0.053^{* * *}$ \\
Unconditional Normal & $(0.010)$ & $(0.017)$ & $(0.008)$ \\
Unconditional Student- $t$ & -0.026 & $0.049^{*}$ & -0.017 \\
& $(0.019)$ & $(0.021)$ & $(0.019)$ \\
Unconditional EVT & $0.033^{* * *}$ & $0.068^{* * *}$ & $0.024^{*}$ \\
& $(0.008)$ & $(0.018)$ & $(0.012)$ \\
& $0.024^{*}$ & $0.089^{* * *}$ & $0.027^{* * *}$ \\
AR-GARCH-Normal & $(0.012)$ & $(0.016)$ & $(0.007)$ \\
AR-GARCH- $t$ & & & \\
AR-GARCH-Normal Filtered HS & & & 0.017 \\
AR-GARCH- $t$ Filtered HS & 0.0112 & $-0.05^{* * *}$ & $(0.023)$ \\
AR-GARCH-Normal FEVT & $(0.020)$ & $(0.013)$ & -0.001 \\
AR-GARCH- $t$ FEVT & 0.012 & -0.033 & $(0.021)$ \\
& $(0.018)$ & $(0.018)$ & 0.022 \\
& $0.037^{* * *}$ & 0.009 & $(0.012)$ \\
& $(0.010)$ & $(0.010)$ & $0.024^{* *}$ \\
& $0.031^{* *}$ & $0.021^{*}$ & $(0.008)$ \\
& $(0.010)$ & $(0.010)$ & $0.089^{*}$ \\
& 0.039 & 0.013 & $(0.047)$ \\
& $(0.038)$ & $(0.033)$ & $0.093^{*}$ \\
& 0.034 & $(0.018$ & $(0.044)$ \\
\hline
\end{tabular}

Notes: This table reports the regression coefficient estimates (and standard errors in parentheses) of regressing the percentage number of VaR violations (obtained using a 1000 day window) on the market capitalization of portfolios. The explanatory variable market capitalization takes values 1 to 10 corresponding to the first to the tenth deciles.

* Significant at $10 \%$

** Significant at 5\%

*** Significant at $1 \%$ 
capitalization to inspect the significance of this variable on the computation of VaR. We perform the regressions using OLS. Table 9 has the estimated regression coefficients and the level of significance of each coefficient for VaR estimates obtained with a window $\omega=1000$. For unconditional methods we observe that there is a significant difference between the number of violations in ten out of twelve cases. VaR models produce higher percentage of violations for larger market capitalization. For the conditional methods, there is a difference in the percentage of violations in seven out of the eighteen cases considered. The conditional model AR-GARCH with Student- $t$ innovations and filtered historical simulation, which is one of the best performing models, produces significantly higher percentage number of violations for larger stocks during crisis periods, non-crisis periods or using the full sample. The filtered EVT model does not reveal a significant difference in the number of violations across different market capitalization portfolios during crises or non-crisis periods but it does if the full sample is used. Note that what most distinguishes this model from the remaining models used is its ability to incorporate returns asymmetry. But different market capitalization portfolios do not reveal very different sample skewness. Hence our results point to the possible existence of different asymmetry characteristics in market capitalization more complex than what sample skewness can measure.

The study is this section infers about differences in number of violations produced by different models by testing if there is a linear relation between number of violations and market capitalization. In the next section we analyze if these differences have an effect on the VaR estimates of portfolios containing different market capitalization stocks. 


\section{VaR of a portfolio with different market capital-}

\section{ization stocks}

The analysis in the previous sections shows that VaR models perform differently for portfolios with different market capitalization. Although these differences are statistically significant it is necessary to show that taking market capitalization into consideration implies better estimates for the VaR of a portfolio containing stocks with different market capitalization.

To answer this question we compare the VaR models performance with and without taking market capitalization into consideration. Suppose that we want to estimate the VaR for the equally weighted portfolio with stocks from all sizes. On the one hand we estimate VaR on the full portfolio returns. On the other hand we estimate VaR for each of the ten market capitalization sub-portfolios and then we calculate the average of these ten VaRs. We consider separately crisis periods, non-crisis periods and the entire sample period. We repeat the exercise with all the VaR methods used previously in this article.

Table 10 has the results for the unconditional VaR methods where VaR was estimated using a window of $\omega=1000$ days. The results using windows of $\omega=500$ and $\omega=250$ are available on request as mentioned before. In the table the column named portfolio corresponds to estimating VaR without taking market capitalization into account and the results in the column average take market capitalization into account. For the entire sample period and for the crisis periods the percentage of violations is always closer to the target VaR confidence level of $1 \%$ when considering market capital- 
ization in the estimation of VaR. For the non-crisis periods three out of four methods perform better without considering market capitalization.

During crisis and non-crisis periods the VaR model performing better is the unconditional EVT for $\omega=1000$ and historical simulation for the other two window sizes. If we use the full sample the student- $t$ model is the best with $\omega=500$ and $\omega=1000$. For $\omega=250$ historical simulation gives better VaR estimates.

The results for the conditional VaR methods, see Table 11, are very significant. Overall for non-crisis periods, crisis periods and full sample considering market capitalization gives better VaR estimates. The models AR-GARCH with Normal or Student- $t$ innovations filtered EVT perform better when market capitalization is taken into account. The two AR-GARCH models with filtered historical simulation perform better in terms of the number of violations when ignoring market capitalization but give superior results in the independence test when considering market capitalization. This pattern is consistent for crisis, non-crisis and full sample periods.

The results are clear, market capitalization has a positive effect on the estimation of portfolio VaR. In principle, estimating VaR for different size sub-portfolios and then aggregate the estimates in a single VaR could be considered a drawback. A proper method for aggregating VaR should imply a multivariate model for the returns on the different sub-portfolios. But we show that even using a simple average gives better VaR estimates than ignoring market capitalization. We would expect that combining market capitalization with a more sophisticated VaR aggregation procedure, adequate for the portfolio at hand, would lead to an even superior performance.

Concerning the best performing models, during crises AR-GARCH- $t$ filtered histor- 
Table 10: Performance of unconditional VaR methods with and without taking into account market capitalization.

\begin{tabular}{|c|c|c|c|c|c|c|}
\hline & \multicolumn{2}{|c|}{ Non-crisis periods } & \multicolumn{2}{|c|}{ Crisis periods } & \multicolumn{2}{|c|}{ Entire sample } \\
\hline & Portfolio & Average & Portfolio & Average & Portfolio & Average \\
\hline \multicolumn{7}{|c|}{ Unconditional historical simulation } \\
\hline$\overline{\mathrm{VaR}}$ & 3.07 & 3.18 & 2.80 & 2.87 & 3.70 & 3.83 \\
\hline$\%$ Viol. & 0.74 & 0.68 & 2.34 & 2.24 & 1.55 & 1.49 \\
\hline $\mathrm{LR}_{\mathrm{uc}}$ & 0.13 & 0.05 & 0.00 & 0.00 & 0.00 & 0.01 \\
\hline $\mathrm{LR}_{\text {ind }}$ & 0.54 & 0.58 & 0.00 & 0.00 & 0.04 & 0.03 \\
\hline $\mathrm{LR}_{\mathrm{cc}}$ & 0.28 & 0.14 & 0.00 & 0.00 & 0.00 & 0.00 \\
\hline \multicolumn{7}{|c|}{ Unconditional Normal } \\
\hline$\overline{\mathrm{VaR}}$ & 2.49 & 2.61 & 2.17 & 2.28 & 3.07 & 3.20 \\
\hline$\%$ Viol. & 1.59 & 1.33 & 4.41 & 3.73 & 2.75 & 2.33 \\
\hline $\mathrm{LR}_{\mathrm{uc}}$ & 0.00 & 0.07 & 0.00 & 0.00 & 0.00 & 0.00 \\
\hline $\mathrm{LR}_{\text {ind }}$ & 0.00 & 0.11 & 0.00 & 0.00 & 0.11 & 0.03 \\
\hline $\mathrm{LR}_{\mathrm{cc}}$ & 0.00 & 0.06 & 0.00 & 0.00 & 0.00 & 0.00 \\
\hline \multicolumn{7}{|c|}{ Unconditional Student- $t$} \\
\hline$\overline{\mathrm{VaR}}$ & 3.17 & 3.29 & 2.70 & 2.82 & 3.82 & 3.97 \\
\hline$\%$ Viol. & 0.81 & 0.64 & 2.46 & 2.30 & 1.55 & 1.46 \\
\hline $\mathrm{LR}_{\mathrm{uc}}$ & 0.27 & 0.03 & 0.00 & 0.00 & 0.00 & 0.01 \\
\hline $\mathrm{LR}_{\text {ind }}$ & 0.51 & 0.60 & 0.00 & 0.00 & 0.04 & 0.03 \\
\hline $\mathrm{LR}_{\mathrm{cc}}$ & 0.44 & 0.09 & 0.00 & 0.00 & 0.00 & 0.00 \\
\hline \multicolumn{7}{|c|}{ Unconditional EVT } \\
\hline$\overline{\mathrm{VaR}}$ & 2.99 & 3.10 & 2.70 & 2.81 & 3.60 & 3.75 \\
\hline$\%$ Viol. & 0.87 & 0.74 & 2.46 & 2.33 & 1.72 & 1.52 \\
\hline $\mathrm{LR}_{\mathrm{uc}}$ & 0.48 & 0.13 & 0.00 & 0.00 & 0.00 & 0.00 \\
\hline $\mathrm{LR}_{\text {ind }}$ & 0.48 & 0.54 & 0.00 & 0.00 & 0.07 & 0.03 \\
\hline $\mathrm{LR}_{\mathrm{cc}}$ & 0.60 & 0.27 & 0.00 & 0.00 & 0.00 & 0.00 \\
\hline
\end{tabular}

Notes: Estimation of VaR, using a 1000 day window, for a portfolio with stocks from all market capitalization sizes. The column portfolio has the estimates without considering size. The column average has the estimates from obtaining VaR for each of the ten portfolios with different market capitalization and then take the average of the ten VaR estimates. $\overline{\mathrm{VaR}}$ denotes the average VaR for the sample period. $\mathrm{LR}_{\mathrm{uc}}, \mathrm{LR}_{\mathrm{ind}}$ and $\mathrm{LR}_{\mathrm{cc}}$ are the p-values of the unconditional coverage, independence and conditional coverage test respectively.

ical simulation using $\omega=1000$ and AR-GARCH- $t$ using $\omega=250$ or $\omega=500$ produce the best VaR estimates. During non-crisis periods the AR-GARCH-Normal filtered EVT with $\omega=250$ and the AR-GARCH-Normal filtered historical simulation with $\omega=500$ or $\omega=1000$ outperform. Using the full sample, the AR-GARCH-t and AR-GARCH- $t$ filtered historical simulation have the best performance for all window sizes. 
Table 11: Performance of conditional VaR methods with and without taking into account market capitalization.

\begin{tabular}{|c|c|c|c|c|c|c|}
\hline & \multicolumn{2}{|c|}{ Non-crisis periods } & \multicolumn{2}{|c|}{ Crisis periods } & \multicolumn{2}{|c|}{ Entire sample } \\
\hline & Portfolio & Average & Portfolio & Average & Portfolio & Average \\
\hline \multicolumn{7}{|c|}{ AR-GARCH-Normal } \\
\hline$\overline{\mathrm{VaR}}$ & 1.97 & 2.05 & 2.69 & 2.81 & 3.00 & 3.09 \\
\hline$\%$ Viol. & 1.88 & 1.52 & 2.46 & 1.75 & 1.65 & 1.36 \\
\hline $\mathrm{LR}_{\mathrm{uc}}$ & 0.00 & 0.00 & 0.00 & 0.00 & 0.00 & 0.05 \\
\hline $\mathrm{LR}_{\text {ind }}$ & 0.13 & 0.22 & 0.80 & 0.84 & 0.18 & 0.27 \\
\hline $\mathrm{LR}_{\mathrm{cc}}$ & 0.00 & 0.01 & 0.00 & 0.00 & 0.00 & 0.08 \\
\hline \multicolumn{7}{|c|}{ AR-GARCH- $t$} \\
\hline$\overline{\mathrm{VaR}}$ & 2.09 & 2.18 & 2.92 & 3.04 & 3.20 & 3.29 \\
\hline$\%$ Viol. & 1.49 & 1.20 & 1.72 & 1.26 & 1.16 & 1.00 \\
\hline $\mathrm{LR}_{\mathrm{uc}}$ & 0.01 & 0.27 & 0.00 & 0.15 & 0.36 & 0.97 \\
\hline $\mathrm{LR}_{\text {ind }}$ & 0.23 & 0.33 & 0.83 & 0.50 & 0.34 & 0.41 \\
\hline $\mathrm{LR}_{\mathrm{cc}}$ & 0.01 & 0.34 & 0.00 & 0.29 & 0.42 & 0.72 \\
\hline \multicolumn{7}{|c|}{ AR-GARCH-Normal Filtered HS } \\
\hline$\overline{\mathrm{VaR}}$ & 2.18 & 2.27 & 3.13 & 3.23 & 3.22 & 3.33 \\
\hline$\%$ Viol. & 1.07 & 0.87 & 1.03 & 0.77 & 0.97 & 0.84 \\
\hline $\mathrm{LR}_{\mathrm{uc}}$ & 0.69 & 0.48 & 0.83 & 0.19 & 0.88 & 0.37 \\
\hline $\mathrm{LR}_{\text {ind }}$ & 0.39 & 0.48 & 0.40 & 0.53 & 0.43 & 0.49 \\
\hline $\mathrm{LR}_{\mathrm{cc}}$ & 0.64 & 0.60 & 0.69 & 0.36 & 0.72 & 0.53 \\
\hline \multicolumn{7}{|c|}{ AR-GARCH- $t$ Filtered HS } \\
\hline$\overline{\mathrm{VaR}}$ & 2.18 & 2.27 & 3.14 & 3.24 & 3.26 & 3.35 \\
\hline$\%$ Viol. & 1.13 & 0.84 & 1.00 & 0.84 & 1.00 & 0.81 \\
\hline $\mathrm{LR}_{\mathrm{uc}}$ & 0.45 & 0.37 & 0.97 & 0.37 & 0.97 & 0.27 \\
\hline $\mathrm{LR}_{\text {ind }}$ & 0.36 & 0.49 & 0.41 & 0.49 & 0.41 & 0.51 \\
\hline $\mathrm{LR}_{\mathrm{cc}}$ & 0.50 & 0.53 & 0.72 & 0.53 & 0.72 & 0.44 \\
\hline \multicolumn{7}{|c|}{ AR-GARCH-Normal Filtered EVT } \\
\hline$\overline{\mathrm{VaR}}$ & 2.05 & 2.15 & 2.73 & 2.78 & 3.13 & 3.21 \\
\hline$\%$ Viol. & 1.75 & 1.42 & 2.33 & 1.88 & 1.26 & 1.10 \\
\hline $\mathrm{LR}_{\mathrm{uc}}$ & 0.00 & 0.02 & 0.00 & 0.00 & 0.15 & 0.56 \\
\hline $\mathrm{LR}_{\text {ind }}$ & 0.16 & 0.63 & 0.11 & 0.82 & 0.31 & 0.37 \\
\hline $\mathrm{LR}_{\mathrm{cc}}$ & 0.00 & 0.07 & 0.00 & 0.00 & 0.21 & 0.57 \\
\hline \multicolumn{7}{|c|}{ AR-GARCH- $t$ Filtered EVT } \\
\hline$\overline{\mathrm{VaR}}$ & 2.02 & 2.12 & 2.72 & 2.78 & 3.11 & 3.20 \\
\hline$\%$ Viol. & 1.97 & 1.46 & 2.33 & 1.97 & 1.36 & 1.10 \\
\hline $\mathrm{LR}_{\mathrm{uc}}$ & 0.00 & 0.01 & 0.00 & 0.00 & 0.05 & 0.56 \\
\hline $\mathrm{LR}_{\text {ind }}$ & 0.48 & 0.66 & 0.11 & 0.48 & 0.27 & 0.37 \\
\hline $\mathrm{LR}_{\mathrm{cc}}$ & 0.00 & 0.05 & 0.00 & 0.00 & 0.08 & 0.57 \\
\hline
\end{tabular}

Notes: Estimation of VaR, using a 1000 day window, for a portfolio with stocks from all market capitalization sizes. The column portfolio has the estimates without considering stock size. The column average lists the VaR estimates computed considering stock size. See also notes in Table 10. 


\section{Conclusion}

Given the recent financial crisis it is obviously necessary to understand better the drivers of financial risk. In this work we explore the role of market capitalization in the estimation of equity VaR. We analyze the performance of different VaR estimation methods for portfolios of stocks with different market capitalization. We consider separately the estimation of VaR during crisis periods, non-crisis periods, and the full sample hence ignoring crises.

Our work reveals that the backtesting procedure without separating crises and non-crisis periods may give misleading results leading to believe that VaR models are performing better than they actually are. Without considering crises and non-crisis periods the resulting good performance can actually be an average of overestimating VaR during non-crisis periods and underestimating VaR during crisis periods. This means that once in a crisis period the regulatory capital will not cover the potentially large portfolio losses. This is important to know not only for risk managers but also for regulators.

We find that VaR methods perform differently for portfolios of stocks with different market capitalization. In the literature, often large market capitalization stocks or indices are used to test VaR models. Hence, we should keep in mind that VaR models performance varies with market capitalization especially when computing VaR for a small number of stocks.

In our study historical simulation methods (unconditional or filtered) give the best performance in several cases. We would expect the EVT method to outperform more 
often than it did especially during crisis periods. This result might be due to the fact that we use data from portfolios containing a large number of stocks. The EVT method will possibly outperform in estimating VaR for individual stocks or portfolios with a small number of assets. The distribution of the returns of a portfolio with many stocks should be typically different from the distribution of an individual stock returns.

We find that taking market capitalization into account produces better VaR estimates. We consider a portfolio with all stocks from small to large market capitalization. Estimating VaR for different size sub-portfolios and then aggregating those VaR estimates gives better backtesting results than computing VaR of the full portfolio returns ignoring market capitalization. Given that financial institutions typically held portfolios with different size stocks, this result has an immediate methodological impact in the risk measurement of portfolios with diverse assets.

It is important to note the consequences, especially during crisis periods, of the fact that taking market capitalization into account improves the performance of VaR methods. During these periods of market instability risk is higher and our results stress the need of understanding the drivers of risk, how they are related, and their effects on existing risk measurement tools.

In this article we concentrate on the effect of market capitalization on the estimation of $\mathrm{VaR}$ for equity portfolios. There is a considerable amount of work to be done exploring the possible roles that economic variables and models in general can play in quantifying and managing financial risk. 


\section{Appendix A}

Recession periods from July 1, 1963 to December 31, 2010 according to the "NBER

Business Cycle Dating Committee" available from

http://www.nber.org/cycles/cyclesmain.html.

\begin{tabular}{llr}
\hline From & - & to \\
\hline December 1969 & - & November 1970 \\
November 1973 & - & March 1975 \\
January 1980 & - & July 1980 \\
July 1981 & - & November 1982 \\
July 1990 & - & March 1991 \\
March 2001 & - & November 2001 \\
December 2007 & - & June 2009 \\
\hline
\end{tabular}




\section{Appendix B}

\section{Conditional AR(1)-GARCH(1,1)-Normal}

Table 12: Performance of Conditional VaR forecasts.

\begin{tabular}{|c|c|c|c|c|c|c|c|c|c|c|c|}
\hline Decile & Lower & 2nd & 3rd & 4 th & 5 th & 6 th & 7 th & 8 th & 9 th & Higher & All \\
\hline \multicolumn{12}{|c|}{ Non-crisis periods } \\
\hline$\overline{\overline{\mathrm{VaR}}}$ & 1.26 & 2.41 & 2.39 & 2.32 & 2.27 & 2.02 & 1.97 & 2.02 & 1.93 & 1.88 & 1.97 \\
\hline$\%$ Viol. & 2.04 & 1.75 & 1.62 & 1.81 & 1.62 & 1.78 & 2.20 & 1.91 & 1.78 & 1.94 & 1.88 \\
\hline $\mathrm{LR}_{\mathrm{uc}}$ & 0.00 & 0.00 & 0.00 & 0.00 & 0.00 & 0.00 & 0.00 & 0.00 & 0.00 & 0.00 & 0.00 \\
\hline $\mathrm{LR}_{\text {ind }}$ & 0.10 & 0.16 & 0.19 & 0.84 & 0.78 & 0.15 & 0.62 & 0.81 & 0.15 & 0.45 & 0.13 \\
\hline $\mathrm{LR}_{\mathrm{cc}}$ & 0.00 & 0.00 & 0.00 & 0.00 & 0.00 & 0.00 & 0.00 & 0.00 & 0.00 & 0.00 & 0.00 \\
\hline \multicolumn{12}{|c|}{ Crisis periods } \\
\hline$\overline{\mathrm{VaR}}$ & 1.66 & 2.72 & 2.85 & 2.91 & 2.99 & 2.89 & 2.95 & 3.01 & 2.97 & 3.12 & 3.69 \\
\hline$\%$ Viol. & 2.53 & 2.33 & 2.49 & 2.20 & 2.23 & 2.27 & 2.30 & 2.14 & 2.20 & 1.81 & 2.46 \\
\hline $\mathrm{LR}_{\mathrm{uc}}$ & 0.00 & 0.00 & 0.00 & 0.00 & 0.00 & 0.00 & 0.00 & 0.00 & 0.00 & 0.00 & 0.00 \\
\hline $\mathrm{LR}_{\text {ind }}$ & 0.00 & 0.00 & 0.43 & 0.65 & 0.07 & 0.00 & 0.72 & 0.60 & 0.65 & 0.84 & 0.80 \\
\hline $\mathrm{LR}_{\mathrm{cc}}$ & 0.00 & 0.00 & 0.00 & 0.00 & 0.00 & 0.00 & 0.00 & 0.00 & 0.00 & 0.00 & 0.00 \\
\hline \multicolumn{12}{|c|}{ Full sample period } \\
\hline$\overline{\mathrm{VaR}}$ & 1.72 & 3.46 & 3.50 & 3.46 & 3.48 & 3.11 & 3.09 & 3.15 & 3.01 & 2.90 & 3.00 \\
\hline$\%$ Viol. & 2.01 & 1.59 & 1.46 & 1.39 & 1.55 & 1.68 & 1.97 & 1.62 & 1.78 & 1.88 & 1.65 \\
\hline $\mathrm{LR}_{\mathrm{uc}}$ & 0.00 & 0.00 & 0.01 & 0.03 & 0.00 & 0.00 & 0.00 & 0.00 & 0.00 & 0.00 & 0.00 \\
\hline $\mathrm{LR}_{\text {ind }}$ & 0.10 & 0.20 & 0.00 & 0.61 & 0.21 & 0.17 & 0.11 & 0.19 & 0.15 & 0.82 & 0.18 \\
\hline $\mathrm{LR}_{\mathrm{cc}}$ & 0.00 & 0.00 & 0.00 & 0.10 & 0.00 & 0.00 & 0.00 & 0.00 & 0.00 & 0.00 & 0.00 \\
\hline
\end{tabular}

Notes: Results from using the conditional AR(1)-GARCH(1,1)-Normal method to estimate the $99 \%$ VaR across the different deciles of market capitalization using a rolling window of 1000 days. $\overline{\mathrm{VaR}}$ denotes the average VaR. The percentage number of violations which fall in the green, yellow and red Basel II color classification have normal, italic and bold type face respectively. $L_{\mathrm{uc}}, \mathrm{LR}_{\mathrm{ind}}$ and $L_{\mathrm{cc}}$ are the p-values of the unconditional coverage, independence and conditional coverage test respectively. 


\section{Conditional AR(1)-GARCH(1,1)-Normal filtered historical sim-}

\section{ulation}

Table 13: Performance of Conditional VaR forecasts.

\begin{tabular}{|c|c|c|c|c|c|c|c|c|c|c|c|}
\hline Decile & Lower & 2nd & $3 \mathrm{rd}$ & 4 th & 5 th & 6 th & 7 th & 8 th & 9 th & Higher & All \\
\hline \multicolumn{12}{|c|}{ Non-crisis periods } \\
\hline$\overline{\mathrm{VaR}}$ & 1.57 & 2.66 & 2.60 & 2.50 & 2.47 & 2.23 & 2.19 & 2.22 & 2.19 & 2.10 & 2.18 \\
\hline$\%$ Viol. & 1.16 & 0.97 & 0.94 & 1.16 & 1.07 & 1.16 & 1.16 & 1.36 & 1.33 & 1.33 & 1.07 \\
\hline $\mathrm{LR}_{\mathrm{uc}}$ & 0.36 & 0.88 & 0.74 & 0.36 & 0.69 & 0.36 & 0.36 & 0.05 & 0.07 & 0.07 & 0.69 \\
\hline $\mathrm{LR}_{\text {ind }}$ & 0.34 & 0.43 & 0.45 & 0.34 & 0.39 & 0.34 & 0.34 & 0.58 & 0.28 & 0.28 & 0.39 \\
\hline $\mathrm{LR}_{\mathrm{cc}}$ & 0.42 & 0.72 & 0.71 & 0.42 & 0.64 & 0.42 & 0.42 & 0.13 & 0.12 & 0.12 & 0.64 \\
\hline \multicolumn{12}{|c|}{ Crisis periods } \\
\hline$\overline{\mathrm{VaR}}$ & 2.05 & 3.22 & 3.29 & 3.35 & 3.47 & 3.29 & 3.45 & 3.36 & 3.37 & 3.41 & 3.13 \\
\hline$\%$ Viol. & 1.20 & 1.03 & 1.13 & 1.03 & 1.90 & 1.10 & 1.16 & 1.20 & 1.13 & 1.20 & 1.03 \\
\hline $\mathrm{LR}_{\mathrm{uc}}$ & 0.27 & 0.83 & 0.45 & 0.83 & 0.60 & 0.56 & 0.36 & 0.27 & 0.45 & 0.27 & 0.83 \\
\hline $\mathrm{LR}_{\text {ind }}$ & 0.33 & 0.40 & 0.36 & 0.40 & 0.46 & 0.37 & 0.34 & 0.33 & 0.36 & 0.45 & 0.40 \\
\hline $\mathrm{LR}_{\mathrm{cc}}$ & 0.34 & 0.69 & 0.50 & 0.69 & 0.67 & 0.57 & 0.42 & 0.34 & 0.50 & 0.42 & 0.69 \\
\hline \multicolumn{12}{|c|}{ Full sample period } \\
\hline$\overline{\mathrm{VaR}}$ & 2.04 & 3.76 & 3.71 & 3.60 & 3.66 & 3.32 & 3.41 & 3.36 & 3.29 & 3.12 & 3.22 \\
\hline$\%$ Viol. & 1.29 & 1.03 & 1.03 & 1.10 & 1.00 & 1.23 & 1.20 & 1.33 & 1.23 & 1.33 & 0.97 \\
\hline $\mathrm{LR}_{\mathrm{uc}}$ & 0.11 & 0.83 & 0.83 & 0.56 & 0.97 & 0.20 & 0.27 & 0.07 & 0.20 & 0.07 & 0.88 \\
\hline $\mathrm{LR}_{\text {ind }}$ & 0.29 & 0.40 & 0.00 & 0.36 & 0.41 & 0.32 & 0.33 & 0.28 & 0.32 & 0.55 & 0.43 \\
\hline $\mathrm{LR}_{\mathrm{cc}}$ & 0.16 & 0.69 & 0.00 & 0.57 & 0.72 & 0.27 & 0.34 & 0.12 & 0.27 & 0.18 & 0.72 \\
\hline
\end{tabular}

Notes: Results from using the conditional AR(1)-GARCH(1,1)-Normal filtered historical simulation method to estimate the $99 \%$ VaR across the different deciles of market capitalization using a rolling window of 1000 days. See also notes in Table 12. 


\section{Conditional AR(1)-GARCH(1,1)-Normal filtered EVT}

Table 14: Performance of Conditional VaR forecasts.

\begin{tabular}{|c|c|c|c|c|c|c|c|c|c|c|c|}
\hline Decile & Lower & 2nd & $3 \mathrm{rd}$ & 4 th & 5 th & 6 th & 7 th & 8 th & 9 th & Higher & All \\
\hline \multicolumn{12}{|c|}{ Non-crisis periods } \\
\hline$\overline{\mathrm{VaR}}$ & 1.30 & 2.53 & 2.65 & 2.47 & 2.37 & 2.11 & 2.07 & 2.18 & 1.95 & 1.87 & 2.05 \\
\hline$\%$ Viol. & 2.27 & 1.75 & 1.23 & 1.65 & 1.85 & 1.85 & 1.78 & 1.62 & 2.14 & 2.43 & 1.75 \\
\hline $\mathrm{LR}_{\mathrm{uc}}$ & 0.00 & 0.00 & 0.20 & 0.00 & 0.00 & 0.00 & 0.00 & 0.00 & 0.00 & 0.00 & 0.00 \\
\hline $\mathrm{LR}_{\text {ind }}$ & 0.70 & 0.16 & 0.48 & 0.80 & 0.84 & 0.39 & 0.15 & 0.78 & 0.22 & 0.14 & 0.16 \\
\hline $\mathrm{LR}_{\mathrm{cc}}$ & 0.00 & 0.00 & 0.35 & 0.00 & 0.00 & 0.00 & 0.00 & 0.00 & 0.00 & 0.00 & 0.00 \\
\hline \multicolumn{12}{|c|}{ Crisis periods } \\
\hline$\overline{\mathrm{VaR}}$ & 1.64 & 2.69 & 2.88 & 2.98 & 2.98 & 2.85 & 2.95 & 3.13 & 2.76 & 2.91 & 2.73 \\
\hline$\%$ Viol. & 2.46 & 2.59 & 2.53 & 2.01 & 2.33 & 2.46 & 2.33 & 1.94 & 2.88 & 2.69 & 2.33 \\
\hline $\mathrm{LR}_{\mathrm{uc}}$ & 0.00 & 0.00 & 0.00 & 0.00 & 0.00 & 0.00 & 0.00 & 0.00 & 0.00 & 0.00 & 0.00 \\
\hline $\mathrm{LR}_{\text {ind }}$ & 0.45 & 0.50 & 0.41 & 0.75 & 0.33 & 0.41 & 0.74 & 0.14 & 0.71 & 0.32 & 0.11 \\
\hline $\mathrm{LR}_{\mathrm{cc}}$ & 0.00 & 0.00 & 0.00 & 0.00 & 0.00 & 0.00 & 0.00 & 0.00 & 0.00 & 0.00 & 0.00 \\
\hline \multicolumn{12}{|c|}{ Full sample period } \\
\hline$\overline{\mathrm{VaR}}$ & 1.85 & 3.58 & 3.78 & 3.71 & 3.60 & 3.23 & 3.19 & 3.42 & 2.96 & 2.80 & 3.13 \\
\hline$\%$ Viol. & 1.55 & 1.49 & 1.03 & 0.94 & 1.36 & 1.46 & 1.62 & 1.00 & 2.10 & 2.49 & 1.26 \\
\hline $\mathrm{LR}_{\mathrm{uc}}$ & 0.00 & 0.01 & 0.83 & 0.74 & 0.05 & 0.01 & 0.00 & 0.97 & 0.00 & 0.00 & 0.15 \\
\hline $\mathrm{LR}_{\text {ind }}$ & 0.21 & 0.23 & 0.00 & 0.45 & 0.27 & 0.66 & 0.19 & 0.41 & 0.69 & 0.43 & 0.31 \\
\hline $\mathrm{LR}_{\mathrm{cc}}$ & 0.00 & 0.01 & 0.00 & 0.71 & 0.08 & 0.05 & 0.00 & 0.42 & 0.00 & 0.00 & 0.21 \\
\hline
\end{tabular}

Notes: Results from using the conditional AR(1)-GARCH(1,1)-Normal filtered EVT method to estimate the $99 \%$ VaR across the different deciles of market capitalization using a rolling window of 1000 days. See also notes in Table 12. 


\section{References}

Andersen, T. G., Bollerslev, T., Christoffersen, P. F., and Diebold, F. X. (2012). Financial risk measurement for financial risk management. Working paper no. 18084, NBER.

Banz, R. W. (1981). The relationship between return and market value of common stocks. Journal of Financial Economics, 9:3-18.

Bao, Y., Lee, T. H., and Saltoğlu, B. (2003). A test for density forecast comparison with applications to risk management. Working paper, Department of Economics, UC Riverside, CA.

Bao, Y., Lee, T. H., and Saltoğlu, B. (2006). Evaluating predictive performance of Value-at-Risk models in emerging markets: A reality check. Journal of Forecasting, 25(2):101-128.

Barone-Adesi, G., Bourgoin, F., and Giannopoulos, K. (1998). Don’t look back. Risk, 11(August):100-104.

Barone-Adesi, G., Giannopoulos, K., and Vosper, L. (1999). VaR without correlations for nonlinear portfolios. Journal of Futures Markets, 19(April):583-602.

Basel Committee on Banking Supervision (1996). Supervisory framework for the use of "backtesting" in conjunction with the internal models approach to market risk capital requirements. Technical report, Available at http://www.bis.org.

Bloom, N., Floetotto, M., and Jaimovich, N. (2009). Really uncertain business cycles. Manuscript, Stanford University.

Bollerslev, T. (1986). Generalized Autoregressive Conditional Heteroskedasticity. Journal of Econometrics, 31:307-327.

Bollerslev, T. and Wooldridge, J. M. (1992). Quasi-maximum likelihood estimation and inference in dynamic models with timt varying covariances. Econometric Reviews, 11:143-172.

Boudoukh, J., Richardson, M., and Whitelaw, R. (1998). The best of both worlds. Risk, 11(May):64-67.

Brooks, C., Clare, A. D., Dalle Molle, J. W., and Persand, G. (2005). A comparison of extreme value theory approaches for determining Value at Risk. Journal of Empirical Finance, 12:339-352.

Chavez-Demoulin, V., Davidson, A. C., and McNeil, A. (2005). Estimating Value-atRisk: a point process approach. Quantitative Finance, 5(2):227-234.

Chavez-Demoulin, V., Embrechts, P., and Sardy, S. (2011). Extreme-quantile tracking for financial time series. Forthcoming in Journal of Econometrics. 
Chernozhukov, V. and Umantsev, L. (2001). Conditional Value-at-Risk: Aspects of modeling and estimation. Empirical Economics, 26(1):271-293.

Christoffersen, P. F. (1998). Evaluating interval forecasts. International Economic Review, 39:841-862.

Christoffersen, P. F. (2012). Elements of Financial Risk Management. Academic Press, Amsterdam, second edition.

Embrechts, P., Klüppelberg, C., and Mikosch, T. (1997). Modelling Extremal Events for Insurance and Finance. Springer-Verlag, Berlin.

Engle, R. and Manganelli, S. (2004). CAViaR: Conditional Autoregressive Value-atRisk by Regression Quantiles. Journal of Business and Economic Statistics, 22:367381.

Fama, E. F. and French, K. R. (1992). The cross-section of expected stock returns. Journal of Finance, 47(2):427-65.

Halbleib, R. and Pohlmeier, W. (2012). Improving the value at risk forecasts: Theory and evidence from the fianncial crisis. Journal of Economic Dynamics 8 Control, $36: 1212-1228$.

Kuester, K., Mittnik, S., and Paolella, M. S. (2006). Value-at-Risk prediction: a comparison of alternative strategies. Journal of Financial Econometrics, 4(1):53-89.

Mancini, L. and Trojani, F. (2011). Robust Value at Risk prediction. Journal of Financial Econometrics, 9:281-313.

McNeil, A. and Frey, R. (2000). Estimation of tail-related risk measures for heteroscedastic financial time series: an extreme value approach. Journal of Empirical Finance, 7:271-300.

Pickands, J. (1975). Statistical inference using extreme order statistics. The Annals of Statistics, 3:119-31.

Pritsker, M. (1997). Evaluating Value at Risk methodologies: Accuracy versus computational time. Journal of Financial Services Research, 12:201-242.

Pritsker, M. (2006). The hidden dangers of historical simulation. Journal of Banking \& Finance, 30:561-582.

RiskMetrics (1996). Technical document. Morgan Guaranty Trust Company of New York.

Rosenberg, B., Reid, K., and Lanstein, R. (1985). Persuasive evidence of market inneficiency. Journal of Portfolio Management, 11:9-17.

Schwert, G. W. (1989). Why does stock market volatility change over time? Journal of Finance, 44:1115-1153. 
Stattman, D. (1980). Book values and stock returns. The Chicago MBA: A Journal of Selected Papers, 4:25-45. 